\title{
CLINICAL QUANTITATIVE SUSCEPTIBILITY MAPPING (QSM) - BIOMETAL IMAGING AND ITS EMERGING ROLES IN PATIENT CARE
}
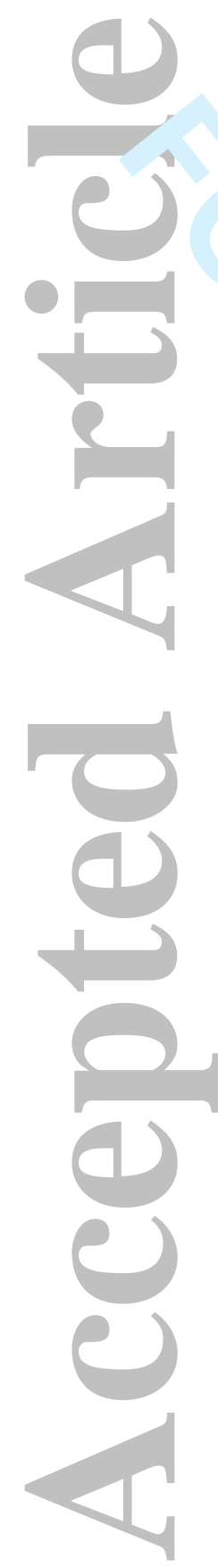

This is the author manuscript accepted for publication and has undergone full peer review but has not been through the copyediting, typesetting, pagination and proofreading process, which may lead to differences between this version and the Version record. Please cite this article as doi:10.1002/ jmri.25693. 


\section{ABSTRACT}

Quantitative susceptibility mapping (QSM) has enabled MRI of tissue magnetic susceptibility to advance from simple qualitative detection of hypointense blooming artifacts to precise quantitative measurement of spatial biodistributions. QSM technology may be regarded to be sufficiently developed and validated to warrant wide dissemination for clinical applications of imaging isotropic susceptibility, which is dominated by metals in tissue, including iron and calcium. These biometals are highly regulated as vital participants in normal cellular biochemistry, and their dysregulations are manifested in a variety of pathologic processes. Therefore, QSM can be used to assess important tissue functions and disease. To facilitate QSM clinical translation, this review aims to organize pertinent information for implementing a robust automated QSM technique in routine MRI practice and to summarize available knowledge on diseases for which QSM can be used to improve patient care. In brief, QSM can be generated with postprocessing whenever gradient echo MRI is performed. QSM can be useful for diseases that involve neurodegeneration, inflammation, hemorrhage, abnormal oxygen consumption, substantial alterations in highly paramagnetic cellular iron, bone mineralization, or pathologic calcification; and for all disorders in which MRI diagnosis or surveillance requires contrast agent injection. Clinicians may consider integrating QSM into their routine imaging practices by including gradient echo sequences in all relevant MRI protocols. 


\section{INTRODUCTION}

Quantitative susceptibility mapping (QSM) solves the deconvolution or inverse problem from magnetic field to susceptibility source to map a local tissue magnetic property $(1,2)$. This local property is fundamentally different from the nonlocal property of traditional gradient echo (GRE) MRI, including susceptibility weighted imaging (SWI), the closely related GRE magnitude T2*_ weighted imaging $(\mathrm{T} 2 * \mathrm{w})$, and GRE phase imaging (Phase), although both QSM and traditional GRE MRI are regarded as being sensitive to susceptibility (3-5). Without deconvolution, traditional GRE MRI generally suffers from blooming artifacts, which 1) may generate contrasts at neighboring locations without susceptibility sources, in addition to at locations with susceptibility sources; 2) strongly depend on imaging parameters, including field strength, voxel size and echo time; and 3) deceptively vary with object orientations, where tissue interfaces with susceptibility differences perpendicular to the main field B0 have much greater contrasts than interfaces parallel to B0 (6). With deconvolution, QSM eliminates the problem of blooming artifacts and provides quantitative distribution of susceptibility sources in tissue. Without deconvolution, traditional GRE MRI can only detect the presence of susceptibility interfaces perpendicular to B0, and cannot localize or quantify any susceptibility source. With deconvolution, QSM can precisely localize and quantify these sources.

The long-standing desire to determine susceptibility sources in tissue arose in the early days of MRI (7). Despite this, the quest to quantify susceptibility as an inverse problem may not have begun in earnest until 2001 (8). Early efforts did not lead to successful susceptibility mapping (912), because they failed to identify additional information needed to solve the ill-posed field-to- 
source inverse problem. A major technological breakthrough came in 2008 when the Bayesian inference with a morphological prior was introduced to form the foundation for QSM (1,13-15). Bayesian inference is a statistical method to optimally estimate susceptibility from both field data that is noisy and incomplete and tissue structure information that also has its uncertainty. Since 2008, research efforts to develop the details of the Bayesian QSM approach have mushroomed, including robust field extraction from MRI signal and effective morphological regularization (6,16-36). The tremendous QSM development efforts in the past 8 years, as evidenced by an exponential growth in the number of QSM papers, have propelled QSM technology from basic research to adaptation and investigation for clinical applications.

QSM accurately maps strong isotropic susceptibility sources in human tissue - predominantly biometals that are highly paramagnetic (mainly iron in ferritin or deoxygenated heme) or present in high concentrations (mainly calcium in mineralization or calcification). QSM of biometals has been valuable in studying disease processes. QSM is shown to be reproducible across scanner makers, models, field strengths, and sites (37-40). QSM can be automated, making it ready for wide dissemination to evaluate its diagnostic and therapeutic value in clinical practice. This will enable clinical investigations both longitudinally and across-centers, ushering in a new era of clinical QSM applications.

QSM can be used to study susceptibility sources other than biometals, particularly white matter (WM) fibers with anisotropic susceptibilities (17). However, anisotropic susceptibility imaging may require much more technical development to overcome the requirement of multiple orientations before it can be applied in clinical studies $(29,41)$. Since most other susceptibility 
sources in human tissue are much weaker than the dominant biometals, we choose to focus on biometal QSM for timely and promising clinical QSM developments, while emphasizing the connection between pathogenic biometals and patient care that is beyond the reach of conventional MRI. We aim to provide readers with basic information on how to 1) implement a robust and automated QSM in their practice, 2) understand the roles of biometals in human health and diseases, and 3) use QSM measurements of biometals in clinical applications.

\section{ROBUST AND AUTOMATED QSM}

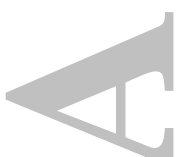

In this technical section on QSM, we aim to provide a conceptual appreciation of the principles of robust QSM based on the Bayesian approach. For integration into daily clinical workflows, we describe an automated QSM that can be implemented across a wide range of major MRI manufacturers, including GE, Philips and Siemens, at both 1.5 and 3 Tesla. The automation and standardization in implementing QSM for biometal imaging is fortunately made possible by the results from rich variations in the Bayesian approach (2).

\section{Fundamental principles of robust QSM}

The main idea underlying QSM is to extract the susceptibility source from its blooming artifacts on traditional GRE MRI through rigorous biophysical modeling of the MRI signal phase. Phase has historically been largely discarded in routine MRI practice, though MRI data is inherently complex, consisting of half phase and half magnitude. Yet, phase data provides rich insight into tissue properties that are complementary to magnitude data (42). Recalling that signal in clinical MRI comes from water (and sometimes fat) protons, phase reflects the inhomogeneous magnetic 
field experienced by protons. The field sources consist of tissue molecular electron clouds and background sources outside tissue. They become magnetized in the MRI main field B0 according to their magnetic susceptibilities and contribute to the magnetic field as dipoles according to Maxwell's equation. The tissue field and background field can be separated according to their source location difference (background field removal). Therefore, MRI phase can be processed to generate the tissue field, which can be analyzed according to the dipole field model to determine tissue magnetic susceptibility (Fig.1).

The magnetic field at a location is the sum of contributions from all surrounding dipole sources. Mathematically speaking, the field is a convolution between the susceptibility spatial distribution and the field of a unit dipole (dipole kernel). Consequently, the determination of tissue susceptibility requires deconvolution of the tissue field with the dipole kernel. Deconvolution in image space is division in k-space (the Fourier convolution theorem). The challenge for this dipole kernel division is that the dipole kernel is zero when an observation point relative to the dipole source is at $\pm 54.7^{\circ}$ (magic angles) with respect to the B0 direction. The observed field contribution at the magic angles should be zero, but there is always noise in the measured data. The resulting division-by-zero of noise (and other data deviation from the dipole field type) leads to streaking artifacts along the magic angles in k-space.

These streaking artifacts are cone-like surfaces distinct from tissue surfaces, manifesting as prominent lines in image space along the complementary magic angles in the sagittal and coronal views and rings in the axial view. Early efforts in solving the field-to-susceptibility inverse problem were not effective in identifying and minimizing streaking artifacts; in fact, the 
truncated $\mathrm{k}$-space division method amplified the streaking artifacts by increasing the deviation from the dipole field type $(12,20)$. The Bayesian approach enables robust suppression of streaking artifacts by tenaciously searching for a solution of minimal streaking $(1,14,15)$. Mathematically, minimal streaking is characterized by penalizing interfaces distinct from tissue interfaces depicted on an anatomic MRI during the search for a susceptibility distribution that satisfies the measured field data. Both noise in the field data and uncertainty in the definition of tissue interfaces are considered in a balanced manner (discrepancy principle) during this tenacious search or numerical optimization, which is termed "Bayesian machine learning" in signal processing or data science (43). While this Bayesian reconstruction is robust (convex optimization), its computation is much costlier than Fourier transform in standard MRI reconstructions. Fortunately, modern numerical optimization tools have allowed the search to be completed within a few minutes on a reasonably equipped desktop computer, now enabling robust QSM in a clinical setting.

\section{Automated QSM processing}

Until a commercial product is available to automatically generate QSM, we recommend the following steps to implement automated QSM on the major scanners for clinical investigations: QSM can be regarded as a postprocessing technique for GRE MRI. The most important factor for enabling QSM is to save faithfully the complex data (both real and imaginary parts, or both magnitude and phase parts) acquired by a GRE MRI, particularly without adulteration of the phase data. 
Once QSM protocols are setup on the scanner to produce these images in DICOM format, a technologist, or ideally an automated image management program on the scanner, can forward these images to a dedicated DICOM image server that is listening for incoming GRE images, from which it reconstructs the QSM images and sends them back to the scanner. The process is automatic and is usually completed within 5 to 10 minutes depending on the computing performance of the server, the connection bandwidth between the scanner and the server, and the matrix size of the GRE data. The advantage of using DICOM is that it is available on all scanner platforms, does not require installation of extra software on the scanner, and has high quality open source implementations.

\section{Brain QSM: data acquisition}

Brain QSM can be well automated. A 3D multi-echo GRE sequence with flow compensation and unipolar readout gradient can be used to image the whole brain. Parallel imaging with properly reconstructed magnitude and phase images can be turned on to reduce scan time $(\mathrm{R}=2)$. The brain region is automatically segmented (44), and the top 1/3 of all edges may be regarded as tissue edges $(2,30)$. A high resolution whole brain imaging of 6-12 mins can be implemented on almost any 3 T scanner with the following parameters: \# of echoes: 8-12; TE: TE1 minimal, $\Delta \mathrm{TE}=3 \mathrm{msec}$; TR: Minimum allowed (typically 50-60 msec); Flip angle: 20; Bandwidth: 400 Hz/pixel; FOV: $24 \mathrm{~cm}$; Slice Thickness: 1-2mm (further halved with zero interpretation in reconstruction, ZIP); Matrix: 400x300x(88-176).

\section{Body QSM: data acquisition}


In contrast to brain QSM, body QSM is less well automated. Body data acquisition must consider respiratory and cardiac motion. This can be achieved using breath-hold or navigator gating, and additional ECG triggering for the heart. Fat chemical shifts have to be accounted for in estimating the susceptibility field from the input complex data $(45,46)$. A body QSM acquisition protocol of $20 \mathrm{sec}$ breathhold (typically used in liver MRI) can be implemented with the following parameters on a 3T: \# of echoes: 6 ; TE: TE1 minimal, $\Delta \mathrm{TE}=3 \mathrm{msec}$; TR: $15 \mathrm{msec}$; R=2; Flip angle: 15; Bandwidth: 300Hz/pixel; FOV: $30 \mathrm{~cm}$; Slice thickness: 3mm (further halved with ZIP); Matrix: $256 \times 176 \times 26$.

\section{QSM challenges and future developments}

This section has provided only a general conceptual overview of the basic principles of QSM. For a more rigorous account of QSM technology, interested readers are referred to a recent technical QSM review with mathematical details (2). Adopting QSM in a clinical setting is an implementation challenge that requires support from MR manufacturers and engineers, and availability of a workstation. There is plenty of room to optimize QSM techniques for both general and specific applications, including shortening acquisition time, improving fat-water separation, reducing shadow artifacts, and establishing a zero reference. For QSM reconstruction, the Bayesian framework seems sufficiently powerful for further exploitations, for example, zero reference for brain QSM may be easily achieved by an additional L2 regularization enforcing the susceptibility of the cerebrospinal fluid (CSF) in the ventricles to be near zero (47).

\section{BIOMETALS IN HEALTH AND DISEASES}


The clinical utilities of QSM come from bridging molecular pathogenesis with patient management. Accordingly, the susceptibility values measured on QSM should be interpreted with the underlying molecular processes in mind. Fortunately, available biophysical knowledge and biomedical data, as reviewed in this section, can help derive sufficient molecular interpretations of QSM in various clinical applications.

\section{Biometals as dominant susceptibility sources - iron, calcification, and contrast agents} The magnetic susceptibilities of various materials are well established (48), with the magnetic periodic table highlighting the strong susceptibility of (ferromagnetic) iron (49). For the human body in the MRI scanner, molecular susceptibility comes primarily from electrons, which have much stronger $\left(\sim 10^{3}\right)$ magnetic moments than protons (42). All orbiting electrons contribute to negative or diamagnetic susceptibility, which is much weaker than $\left(\sim 10^{-2}\right)$ any positive or paramagnetic susceptibility coming from the magnetic moment of an unpaired electron. The major components in a cell consist of water, proteins, lipids, minerals, and carbohydrates (50), and the most abundant elements in the body include oxygen, carbon, hydrogen, nitrogen, calcium, phosphorous, magnesium, potassium, sulfur, sodium, chlorine, iron, and zinc $(51,52)$. According to known material susceptibility values and physical chemistry (48), metallic compounds will dominate susceptibility. Therefore, the high abundance and high susceptibility of cellular iron compounds make iron the major biometal source for tissue QSM.

Of course, the claim that iron is the dominant source for QSM for any given tissue can only be confirmed by biochemical analyses. Immunohistochemistry and mass spectroscopy studies have 
indicated that iron is the dominant high susceptibility biometal stored primarily in ferritin $(28,53$ 55). Very high concentrations of calcium in calcification or mineralization (hydroxyapatite crystal) (50) causes strong negative (diamagnetic) susceptibility. Additionally, contrast agents (gadolinium and iron compounds) at sufficient concentration in clinical and molecular MRI are highly paramagnetic, therefore showing high values on QSM. While our discussion is generalizable to other biometals, we focus here on iron, calcium, and contrast agents.

\section{Systemic and brain iron homeostases: heme, ferritin and labile iron, and QSM sensitivity}

The electronic configuration of iron, [Ar] $3 \mathrm{~d}^{6} 4 \mathrm{~s}^{2}$, makes iron compounds commonly used as catalysts in organic syntheses (56). By converting between ferrous $\left(\mathrm{Fe}^{2+}\right)$ and ferric $\left(\mathrm{Fe}^{3+}\right)$ forms, iron functions as both an electron donor and acceptor with essential roles in human physiology. Approximately $65 \%$ of iron in the body is in the $\mathrm{Fe}^{2+}$ form bound to the hemes of the hemoglobin protein in red blood cells (RBCs) (50), which are involved in oxygen binding and transportation. Oxygen binding cause a strong heme porphyrin-iron interaction that splits the $\mathrm{Fe}^{2+} 3 \mathrm{~d}$ orbit and pairs the 6 residing electrons. Consequently, $\mathrm{Fe}^{2+}$ in oxyheme $(\mathrm{oH})$ loses its strong paramagnetism, and $\mathrm{oH}$ is actually weakly diamagnetic (57). Therefore, only paramagnetic deoxyheme $\mathrm{Fe}^{2+}$ in the veins contributes to the observed QSM values $(58,59)$, enabling QSM to measure blood oxygen levels noninvasively throughout the body.

Other than iron in RBCs, $0.2-3 \%$ of total cellular iron is in the labile iron pool, which is too dilute to show on QSM. Labile iron bioactively participates in cellular biochemistry, including energy metabolism, mitochondrial respiration, lipid synthesis, DNA synthesis, neurotransmitter synthesis, and many other functions (60). Though labile iron is contained in various shielding 
metalloproteins, it still exhibits the redox property of catalyzing the formation of radicals that can be harmful to nucleic acids, proteins, and lipid membranes (61). Therefore, iron uptake, transport and storage are highly regulated to maintain homeostasis, involving many proteins including hepcidin, ferroportin, transferrin, transferrin receptors (TfR) 1 and 2, divalent metal transporter (DMT)-1, and others $(61,62)$. As the body lacks an effective mechanism to excrete iron, it depends upon rigorous hepcidin regulation of intestinal iron absorption to preserve the iron balance. Consequently, iron deficiency, which can cause anemia and may be easily remedied by an iron supplement, is less a concern as compared to iron overload, both cerebral and systemic, which can cause extensive tissue damage (62).

Systemic iron homeostasis is preserved by efficient use of iron for production of RBCs, prompt recycling of iron from hemoglobin in RBCs at the end of their lifespan, rigorous regulation of iron storage within macrophages and hepatocytes, and meticulous control of intestinal iron absorption (Fig.2a) (62). Brain iron homeostasis is separated from systemic iron homeostasis by the blood-brain barrier (BBB) and CSF barrier. Blood plasma iron may enter the brain through brain capillary endothelium and choroid plexus epithelium (Fig.2b) (63). The mechanisms to maintain iron supply to neurons, oligodendrocytes, microglia, and astrocytes are incompletely understood. There are overlapping, but not identical, proteins and mechanisms between systemic and brain iron handling (64), with transferrin as a major vehicle to diffuse through the cerebrospinal and interstitial fluids for delivering iron to brain cells expressing TfR.

The vast majority $(>97 \%)$ of iron in cells is stored as $\mathrm{Fe}^{3+}$ (ferrihydrite) in the spherical shell protein ferritin, readily available for conversion into the labile iron pool (60) and measurable on 
QSM (28). The ferritin iron concentration is in a homeostasis-determined equilibrium with the labile iron concentration (65). In pathological conditions, the amount of iron may exceed the storage capability of cellular ferritin. The excess may be stored as $\mathrm{Fe}^{3+}$ in other proteins such as neuromelanin in dopaminergic neurons (66), hemosiderin in chronic hemorrhage (67) and hemosiderin in tissue iron overload (68), becoming poorly available for conversion into the labile iron pool. $\mathrm{Fe}^{3+}$ also accumulates as magnetite in the characteristic amyloid plaque pathology of Alzheimer's disease (69). It is assumed that the stored ferric iron $\left(\mathrm{Fe}^{3+}\right)$ has the same paramagnetic susceptibility of 5 unpaired electrons, but this remains to be proven. This highly paramagnetic $\mathrm{Fe}^{3+}$ contributes to the high QSM values observed in the nuclei of the midbrain and subcortical gray matter in all brains, which require high iron levels for generation of neurotransmitters.

\section{Brain iron overload in neurodegeneration}

Brain iron overload (more than that can be safely transported and stored) is a cause and/or cofactor of a variety of neurodegenerative diseases, including Alzheimer's disease (AD), Parkinson's disease (PD), Huntington's disease (HD), Friedreich's ataxia (FA), and amyotrophic lateral sclerosis (ALS) (70). Iron overload leads to oxidative stress, damaging cellular contents including proteins and mitochondria, and causes inflammatory toxicity (Fig.3). These features are common to neurodegenerative brains but with iron overload locations varying with diseases.

PD, which affects $\sim 1 \mathrm{M}$ Americans (71), is defined pathologically by the loss of dopaminergic neurons in the substantia nigra pars compacta (SNc) (72). Motor symptoms associated with disruption of dopamine release include resting tremor, rigidity, bradykinesia, and postural 
instability (73). The selective SNc neurodegeneration is not yet understood but possibly occurs as a result of a complex interplay of aging, genetic susceptibility, environmental factors, and prooxidant iron accumulation in the SNc (74). The SNc is rich in iron (75), and levels only increase with age (76). Pathology of PD brain tissue has consistently established elevated iron in the SNc (77). Noninvasive MRI of PD has demonstrated that nigral iron increase correlates with disease severity (78), duration (79), and longitudinal progression (80). Possible nigral iron elevation pathways include increase of TfR2 iron import (81), elevation of the DMT1 (82), and failure of iron export (83). Elevation in nigral iron leads to oxidative stress, increasing lipid peroxidation (84), reducing glutathione levels (85), damaging DNA (86), accelerating the aggregation of $\alpha$ synuclein (87), and causing mitochondria dysfunction (88). Iron elevation in PD can also cause pro-inflammatory microglia activation (89) to contribute to neurodegeneration (Fig.3)(90).

\section{Systemic iron overload}

Iron overload occurs with hepcidin deficiency or ferroportin resistance to hepcidin (91) in various diseases, including hemochromatosis, alcohol-related liver disease, and chronic transfusion refractory anemia. The liver is the only organ whose iron content is invariably increased in all forms of systemic iron overload (92). Excess iron is present both in reticuloendothelial macrophages (Kupffer cells) and in parenchymal cells (hepatocytes) with a spatial distribution dependent upon the underlying disorder (93). With increasing body iron, hepatocytes may eventually exhaust the capacity to safely store the excess iron. Consequent oxidative damage to hepatocytes causes paracrine induction of hepatic stellate cells and portal myofibroblasts, resulting in collagen deposition, fibrosis, micronodular cirrhosis and, finally, hepatocellular carcinoma (94) 


\section{Iron in inflammation}

Iron is vital to invading pathogens, as microbes use iron for pathogen proliferation, virulence, and persistence (95). The immune response involves iron utilization by the host and iron restriction to pathogens (Fig.4). Host and pathogens compete for control over iron homeostasis to influence the course of an infectious disease (96). In innate immunity, macrophages play critical roles through various activations ranging from pro-inflammatory (M1) to anti-inflammatory (M2) (96). M1 macrophages release toxins including reactive oxygen and nitrogen species and deplete iron from the environment to limit the iron-supply to suspected pathogens (Fig.4a). M2 macrophages recycle and release iron into the environment to repair tissue (Fig.4b), clear cellular debris, and remodel surrounding matrices (96). Macrophages have several uptake processes, including through TfR1, DMT1, lactoferrin receptor, heme or hemoglobin scavenger receptor, and erythrophagocytosis; macrophages can export iron only through ferroportin $(96,97)$.

Multple Sclerosis (MS) is an inflammatory disease of the central nervous system (CNS) that affects more than 400,000 Americans (98). The enigmatic pathogenesis of MS includes acute focal demyelination (lesion formation caused by infiltration of $\mathrm{T}$ cells and macrophages, frequently around a vein) and chronic tissue damage $(99,100)$. Chronic active WM lesions have iron enriched M1 microglia (innate brain macrophages) at their rims $(89,101-103)$ that express proinflammatory cytokines and cause persistent tissue damage, contributing to MS disease progression (89). However, we have yet to understand iron trafficking in the entire MS brain, including 1) iron uptake in the basal ganglia $(104,105)$, and 2) iron decrease in normal appearing WM during secondary progression (103). 


\section{Iron in circulating red blood cells}

Oxygen consumption is critical for oxidative phosphorylation in aerobic respiration, where cells in the brain, heart, and other organs derive most of their energy (50). The brain represents $\sim 2 \%$ of the adult human body weight, yet it consumes $20 \%$ of the total oxygen supply (50). Consequently, a deficiency in blood oxygen delivery can easily damage brain tissue, as in hypoxia of Alzheimer's disease (AD) (106) and multiple sclerosis (107), and as ischemia in stroke (108). Quantitative mapping of the cerebral metabolic rate of oxygen consumption (CMRO2) is especially valuable for evaluating these brain disorders (109).

Bleeding in the brain or intracerebral hemorrhage ( $\mathrm{ICH})$ is a devastating disease with high mortality (about 40\% at 1 month) (110). Furthermore, microbleeds are increasingly recognized in cerebrovascular diseases, dementia, and normal aging (111). When RBCs leave the vascular space, oHs in RBCs immediately and rapidly become deoxygenated into highly paramagnetic dHs (112). Activated microglia/macrophages start to phagocytose RBCs. RBCs lyse and release degraded hemoglobin called methemoglobin containing $\mathrm{Fe}^{3+}$ (more paramagnetic than $\mathrm{Fe}^{2+}$ ) (113), as well as other molecules such as carbonic anhydrase (114). Heme in methemoglobin is further degraded by heme oxygenase to release iron. Both iron and carbonic anhydrase contribute to neuron injury and death in ICH; corresponding therapies are now in clinical trials (115).

\section{Calcium homeostasis - bone mineralization and calcification}

Compared to iron, calcium is about 300 times more abundant in the human body (52), with a small fraction (1.1\%) in the labile calcium pool ( $1 \%$ is intracellular and $0.1 \%$ extracellular), and 
the vast majority $(98.9 \%)$ stored in bones. Sufficient labile calcium ions $\left(\mathrm{Ca}^{2+}\right)$ are needed for important cellular functions, including signal transduction, muscle contraction, and cell membrane potential. Excessive labile $\mathrm{Ca}^{2+}$ can cause cellular damage, including excitotoxicity, neurodegeneration, and apoptosis. The labile calcium concentration is in equilibrium with bone calcium through release and reabsorption, processes that are tightly regulated by calcitonin/parathyrin hormones from thyroid/parathyroid glands (Fig.5). These hormones also control calcium absorption and secretion in the intestines and calcium filtration and reabsorption in the kidneys. Calcium homeostasis maintains a serum $\mathrm{Ca}^{2+}$ concentration precisely within 1.10 $-1.35 \mathrm{mM}(50) . \mathrm{Ca}^{2+}$ can be bound to phosphate salts in a collagen-proteoglycan matrix to form hydroxyapatite crystals, the process by which bone is mineralized (50). Calcification also occurs in vascular plaques and in apoptotic cells in necrotic tumors (116). Highly-concentrated calcium salts in mineralized bone or calcification form a strong diamagnetic source measurable on QSM.

Disruption in calcium homeostasis can result in a number of pathologic disorders including osteoporosis with decreased bone mass and strength, calcification in atherosclerotic plaques, and calcification in tumors. Osteoporosis is a highly prevalent disorder affecting the older population, particularly Caucasian women after menopause, causing fractures commonly in the hips and forearms (117). While high-grade tumors contain hemorrhages from leaky blood vessels or dysregulated angiogenesis, calcification tends to be found in various low-grade tumors of the bladder, breast, ovaries, brain, lungs, and gastrointestinal tract, though mechanisms of tumor calcification have yet to be fully clarified (118). Calcification is commonly observed in atherosclerotic plaques and may play a positive role in plaque stability (119). 


\section{Contrast agents as exogenous biometals}

To assess vascularity in MRI, contrast agents (CA) are routinely used for detection of micro vessel wall permeability changes associated with cancer, metastasis, and inflammation, and to map macro vessel lumen stenosis and other deformations in various vascular diseases (120). In addition to functioning as an imaging agent aiding in diagnosis, magnetic cores can be incorporated into functionalized nanoparticles delivering therapeutic drugs (121). Most MRI CAs use chelators, macromolecules, or nanoparticles to house gadolinium or iron that is highly paramagnetic with unpaired electrons. The unpaired electronic spins in CA molecules interact with bounded water proton spins, which also interact with surrounding MR-measureable free water proton spins. This interaction among $\mathrm{CA}$ and bound and free $\mathrm{H}_{2} \mathrm{O}$, accelerates the loss of energy and coherence of protons excited by RF, i.e., increases the T1,T2 relaxation rate (R1,R2) (122). The enhancement effect on R1 measured as percent increase is higher than that on R2, because when unenhanced, R2 > R1 (123). Accordingly, T1-weighted imaging is routinely performed in contrast-enhanced MRI, including dynamic contrast-enhanced (DCE) MRI for tissue perfusion imaging (124). CAs also enhance T2* hypointensity (increases in T2* rate, R2*) of the intravoxel dephasing because of the strong dipole field of their unpaired electrons. This CA T2* contrast enhancement is used in dynamic susceptibility contrast (DSC) MRI for tissue perfusion imaging (124) and in tracking cells and other biomedical applications targeted by magnetic nanoparticles (121).

\section{CLINICAL APPLICATIONS ENABLED BY QSM BIOMETAL IMAGING}


QSM can be used in all clinical applications of traditional GRE MRI including T2*w, Phase, and SWI (125). Here, we describe only clinical applications that are enabled by QSM but that are beyond the reach of traditional GRE MRI. Our discussion of clinical applications focuses on using QSM to connect pathogenesis with patient care. There are many diseases for which QSM can be used to measure biometal changes during pathogenesis, progression, and treatment. To illustrate the potential of these clinical applications, we use major cell types, their biometals and generalizable disease examples. Accordingly, this section is organized into iron in neurons, iron in hepatocytes, iron in macrophages/microglia/Kupffer cells, iron in red blood cells, calcification in bone and apoptotic cells, and biometal contrast agents.

\section{Iron in neurons: neurodegenerative diseases exemplified by PD}

The potential of QSM applications in neurodegenerative diseases can be illustrated with PD. Typically, the motor symptoms that afflict PD patients can be controlled initially by medications for 4-6 years and then later require surgical deep brain stimulation (DBS) (Fig.6) or an apomorphine pump (126). Presently, neuroprotective or disease-modifying therapy, such as ironchelating therapy (Fig.7) (127), for PD is not yet available but is under development.

QSM has been actively applied to study the brains of PD patients $(128,129)$, demonstrating greater sensitivity than $\mathrm{R} 2 *$ in identifying increased nigral iron in PD patients as compared to healthy controls (130-135). The voxel-based morphology analysis by Du et al (132) elegantly demonstrates selective SNc iron increase in perfect concordance with post mortem histology, underscoring the potential of QSM as a biophysical marker for therapeutic effects in clinical trials. While these studies may provide little direct value for current symptomatic levodopa 
medication of PD patients, QSM could be an essential imaging tool for DBS and iron-chelating therapy. Recently, cortical regions have also shown to be affected by iron accumulation detected by QSM (136), which is of relevance to the cognitive deterioration that also occurs as a complication as the disorder progresses.

DBS is efficacious in controlling the motor symptoms of advanced PD, including tremors and dyskinesia (137). By introducing electrodes into the subthalamic nucleus (STN), a small ellipsoid with width/height/depth=10/7/3mm (138), DBS delivers electrical stimuli to excite nearby fibers or disrupt aberrant signaling (Fig.6)(137). Since the position of the active contact within the STN is the only variable to predict the outcome of STN stimulation, precise targeting of the STN lateral region within $0.5 \mathrm{~mm}$ is essential for achieving sufficient DBS efficacy (139). Given that the STN actively generates glutamine and is therefore rich with iron, using QSM to locate the STN is advantageous because it provides much superior contrast-to-noise ratio over other MRI methods, including T2 weighted-imaging (T2w), T2*w, R2*, phase, and SWI (Fig.8)(140). Therefore, QSM is an ideal technique to accurately and precisely guide electrode placement in DBS treatment for advanced PD patients and other patients with tremor and dystonia $(140,141)$. Current promising results strongly argue in favor of additional clinical evaluations across multiple DBS centers to evaluate whether the inclusion of QSM in presurgical MRI (rather than current T2w protocols) can overall improve health outcomes in PD patients undergoing DBS.

A great potential QSM application in patient care is to monitor iron-chelating therapy, which plays a vital role in treating patients with iron overload in the liver, heart, and other organs $(142,143)$. Relevant to neurodegenerative diseases is the iron chelator deferiprone (DFP), a small 
lipophilic molecule that can cross the BBB and enter the substantia nigra (144). DFP is approved for use with transfusional iron overload, and is currently in clinical trials for treatment of brain iron accumulation disorders such as PD (clinicaltrials.gov). Devos, et al. have recently reported very encouraging results showing that DFP improves PD motor performance and reduces nigral R2* values on GRE MRI, suggesting that DFP can be the first disease-modifying therapy for PD (127). Because QSM is superior to R2* for evaluating nigral iron $(131,132,134,135,145)$, an important potential application of QSM in PD is to measure DFP's effectiveness of targetengagement in clinical trials.

Iron-chelating therapy is being explored to treat other neurodegenerative diseases (146). QSM has been applied to study iron overload in AD (147-149), HD (150), FA (151) and ALS (152). Given the urgent need for developing disease-modifying therapies for neurodegenerative diseases, QSM may facilitate the development of such novel therapeutic agents.

\section{Iron in macrophages and microglia: inflammatory diseases exemplified by MS}

The potential of QSM applications in inflammatory diseases can be illustrated in MS. Currently, there are several drugs that work to suppress MS inflammation through various mechanisms of action (153). To optimize treatment of MS patients, it is essential to accurately capture CNS inflammatory activity. Current imaging techniques detect accumulation of gadolinium $(\mathrm{Gd})$ in regions of BBB leakage that occur only during acute lesion formation. Unfortunately, the substantial and long-lasting microglial inflammation behind an intact BBB that occurs in established lesions cannot be detected by Gd accumulation $(154,155)$. This limits the ability of 
the treating physician to assess in MS patients whether CNS inflammation has been successfully stopped, or whether it continues with smoldering, low-grade microglial activation.

QSM has been used to study abnormal iron accumulation in various brain regions in MS, including basal ganglia (156,157), cortical gray matter (158-160), and WM lesions $(102,158,161$ 168). Specifically, the QSM-hyperintense rim of a WM lesion corresponds to iron in proinflammatory activated microglia (102) (Fig.9). Consequently, WM lesions with rim iron (persistent phase rims (169) or hyperintense QSM rim (164)) sustain more tissue damage than lesions without rim iron. The magnetic susceptibility of WM lesions, and by inference, ironcontaining microglia, dynamically evolve in MS patients $(161,167)$. Susceptibility is isointense in enhancing (actively demyelinating) lesions (circle in Fig.10a), increases sharply after enhancement subsides (early chronic lesions, arrows in Fig.10a), and then stays constant for an extended period of several years before dissipating into isointense levels (chronic silent lesions). Therefore, QSM provides a wide window into chronic inflammatory activity in established nonenhancing lesions. QSM measurement of chronic inflammation in MS lesions that appear stable on conventional MRI but have a high burden of lesional microglial activation are of significant therapeutic and diagnostic importance. Several compounds that are either FDA approved (Tecfidera) or under development (Laquinimod (170) and Siponimod (171)) have been shown to impact microglial activation $(172,173)$. QSM can be utilized to monitor these therapies for their ability to mitigate iron accumulation in microglia.

In current standard clinical MS MRI protocols, Gd injection is required to differentiate between active (enhancing) and nonactive (nonenhancing) lesions. Since MS patients undergo regular 
imaging, repeated Gd injections put them at risk for Gd accumulation in the brain $(174,175)$, this was reported to be associated with degradation into secondary progression (176). Accordingly, an active area of MS MRI research is to eliminate Gd injection and to reduce scan time and cost (177-179). Because of myelin debris formation and removal, and iron accumulation immediately after the BBB seals (166), enhancing lesions are isointense on QSM while nonenhancing lesions are hyperintense on QSM. This enables QSM to accurately predict the status of the BBB without Gd injection (168) (Fig.10b).

Before initiating expensive medication, it is important to differentiate MS from MS mimicking conditions, including neuromyelitis optica spectrum disorder, systemic autoimmune diseases, cerebral small vessel disease, and migraine (180). An MRI biomarker to differentiate MS from its mimics is the central vein sign (CVS) defined T2* ${ }^{*}$ hypointensity (180). By eliminating TE dependence in $\mathrm{T} 2{ }^{*} \mathrm{w}$ hypointensity, QSM can provide a universal CVS definition (Fig.10c), while other features in QSM including the hyperintense rim may also be explored for differentiating MS from MS mimics. QSM may have a broad utility in other disorders characterized by chronic microglial activation such as AD and systemic lupus erythematosus (181).

\section{Iron in hepatocytes and Kupffer cells: resolve R2/R2* confounders}

Three iron chelating agents, deferoxamine, deferiprone and deferasirox, are available to treat chronic systemic iron overload (143). These chelating agents form a complex with iron, promoting its excretion (Fig.7) by removing excess iron from cells and clearing plasma nontransferrin-bound iron. Iron-chelating therapy requires careful monitoring of tissue iron 
concentrations to avoid adverse effects of excessive chelator administration. R2 and R2* methods based on MRI signal magnitude are used for noninvasively evaluating liver and heart iron concentrations (182-185). However, their accuracy can be limited by confounding factors, including fat, fibrosis, and edema. Both R2 and R2* depend on intravoxel contents in a very complex manner (186), making it very difficult to resolve iron content from these confounders. Fortunately, QSM has a simple linear relationship with these intravoxel contents according to chemical decomposition, allowing linear extraction of iron content. Furthermore, except fat (quantifiable according to its chemical shifts and phase data (45)), other intravoxel contents, including fibrosis and edema, have a susceptibility that is virtually zero relative to water, making QSM ideally suited for determining the iron concentration of liver, heart, and other tissues $(45,187)$ (Fig.11). Therefore, accurate tissue iron measurement enabled by QSM has enormous potential to play an important role in monitoring iron-chelating therapy of iron overload.

\section{Iron in red blood cells: oxygen consumption and hemorrhage}

We first use the brain as an example for using QSM to study oxygen consumption. The ability to perform non-invasive CMRO2 mapping could improve our ability to manage a variety of neurological disorders. For example, the small fraction $(<10 \%)$ of stroke patients undergoing treatment may be increased following identification of salvageable ischemic penumbra and irreversibly damaged core; this is unfortunately not possible with current cerebral blood flow (CBF) (190), diffusion weighted imaging (191), and oxygen extraction fraction (OEF) (192). CMRO2 (=CBF* $[\mathrm{dH}]$, assuming fully oxygenated arterial blood; $[\mathrm{dH}]$ denotes $\mathrm{dH}$ concentration) (193) promises to define the ischemic penumbra-core by providing a direct measure of cell metabolism that consistently predicts neuronal death (194-196). Current CMRO2 mapping 
techniques are too cumbersome to be used even in research, including 1) PET with ${ }^{15} \mathrm{O}$ of a very short 2-min half-life (197), 2) QUIXOTIC MRI with problematic flow capture, poor sensitivity, and arterial and CSF contamination (198,199), 3) quantitative BOLD MRI with model errors and poor-conditioned inversion (200,201), and 4) calibrated fMRI with model errors and two vascular challenges (202-205). Calibrated fMRI has been attempted by several groups, and QSM can simplify its application while eliminating its empirical assumptions. Tissue susceptibility measured on QSM is linearly related to $[\mathrm{dH}]$; in contrast, the $\mathrm{R} 2 *_{-}[\mathrm{dH}]$ model in calibrated fMRI is nonlinear and empirical with extra parameters of unclear physical origins (205). QSM-based CMRO2 mapping can be performed using a simple vascular challenge such as hyperventilation (206) (Fig.12). It may be viable to develop challenge-free CMRO2 mapping by optimally utilizing both MRI signal and biological priors (207). This is fertile ground for technology development of CMRO2 mapping and clinical investigations enabled by practical CMRO2 techniques.

-

The very high susceptibility of ICH presents an opportunity to further develop QSM (208-211). QSM has been used to study intracerebral hemorrhage, including measurement of hematoma volume (212), differentiation from calcification $(18,213,214)$, and dating of cerebral cavernous malformation lesions (215). Preventing hematoma growth is an important goal in improving patient outcomes as well as an important endpoint in clinical trials focused on the treatment of acute $\mathrm{ICH}$ (216). By overcoming blooming artifacts, QSM can be used to measure hematoma volume as accurately as CT, while providing MRI benefits of assessing tissue damage. Treatments to minimize neuron damage from $\mathrm{ICH}$ are under development and include iron- 
chelating therapy (217). QSM would be an ideal method for evaluating iron levels in emerging ICH therapies.

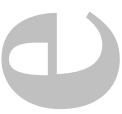

QSM can also be used to measure the burden of cerebral microbleeds (CMB) (218), which is a strong and independent risk factor for anticoagulant-associated ICH (219). In general, the longterm clinical risks and management in patients with microbleeds have yet to be defined. This remains an active area of investigation, where QSM can play an important role, particularly in longitudinal studies of microbleed burden (220).

QSM can also be applied to circulation (221). Chamber blood oxygenation, which is evaluated in deciding surgery for intracardiac shunt in congenital heart disorders (50), may be directly quantified by QSM, avoiding invasive oximetry. QSM may be developed to quantify intramyocardial hemorrhage (IMH), which often follows revascularization of myocardial ischemia (222) and shares features with hemorrhagic transform in ICH (223).

\section{Iron deficiency}

Lastly, QSM can be used to study brain iron deficiency in children (188), in adults with restless leg syndrome (189), and, potentially, to detect iron deficiency in the bone marrow, liver and other organs. Restless leg syndrome (RLS; Willis-Ekbom disease), characterized by an irresistible urge to move the legs, is a neurosensorimotor disorder associated with iron deficiency, with iron levels decreased in the substantia nigra, thalamus, putamen, and pallidum. QSM may offer a new reference means for non-invasive detection of iron deficiency that can avoid the 
confounding effects of inflammation, infection, and malignancy on currently available biomarkers.

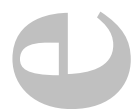

\section{Bone mineralization and pathologic calcification}

Osteoporosis can be treated through medications, including anti-resorptive agents

(biophosphonates) and anabolic agents that slow down disease progression and reduce fracture risk (224). Osteoporosis is diagnosed on measurements of bone mineral density (BMD) using dual energy x-ray absorptiometry (DXA) and quantitative computed tomography (QCT) (225).

3D QCT is superior to 2D DXA in measuring trabecular and spinal bones that are more sensitive to therapy (226). However, QCT is limited in usage, because of its much higher radiation dose than DXA. This problem can be solved by the latest development of bone QSM which tomographically quantifies calcium without radiation (227) (Fig.13). Because of the widespread use of bone mineral densitometry as a screening tool for osteoporosis, there is the potential for significant public health benefit if the radiation dose is minimized by using MRI QSM instead of X-rays.

QSM has been used to study calcification in tumors $(2,228)$ and to resolve hemorrhage from calcification; their distinction mapped on QSM may indicate tumor malignancy. QSM of arterial calcification could have an emerging role in quantifying calcification in arterial beds, including in the coronary arteries where calcium scores have been shown to be a highly predictive measure of overall cardiovascular risk. Among patients with significant plaques, using QSM to differentiate calcium from hemorrhage may great clinical impact because intraplaque 
hemorrhage is a potential trigger of plaque vulnerability (229) while calcification may be an indicator for plaque stability (119).

\section{Contrast agent (CA) biodistribution}

Historically, QSM started with the curiosity to quantify Gd in contrast-enhanced magnetic resonance angiography from the change in the phase images that were and still are discarded in routine MRI (230,231). In current clinical MRI, CA biodistribution quantification for mapping tissue perfusion is obtained by assuming that CA concentration ([CA]) is linearly proportional to signal change in DCE or DSC MRI (123). This assumption may break down in tumors where [CA] is elevated due to highly active angiogenesis. In general, absolute quantification of [CA] according to its $\mathrm{T} 1 / \mathrm{T} 2$ effects requires calibration, is susceptible to flip angle errors, and relies on the problematic empirical assumption that the change in the $\mathrm{T} 1 / \mathrm{T} 2$ relaxation rate is linearly proportional to [CA]. The linearity assumption requires sufficient availability of bulk water, which is not true for localized CAs with limited access to water, as demonstrated in the wellknown T1/T2 relaxation quench in molecular MRI (232). T2* hypointensity or R2* is notoriously difficult to quantify and suffers from saturation and blooming artifacts. QSM can overcome these problems associated with R1, R2 and R2* for [CA] quantification $(233,234)$. In QSM, the CA susceptibility field is measured by its neighboring water, without requiring water

contact, thus overcoming the quench problem. QSM also overcomes the R2* saturation problem using distant water, and of course overcomes R2* blooming artifacts through deconvolution. QSM may play an important role for quantifying drug biodistribution, with major potential implications for improving drug delivery. 


\section{SUMMARY}

In this clinical QSM review, we have described QSM as a robust and automated technique to image disease-related biometals with strong susceptibility values, especially iron and calcium. As examples of applications related to iron in neurons, QSM provides excellent definition of the subthalamic nulei to accurately guide deep brain stimulation in patients suffering from Parkinson's disease. QSM holds great promise for monitoring iron-chelating therapy, which is used in treating iron overload in the liver, heart and other tissues and is actively being investigated for treating neurodegenerative diseases with iron overload and hemorrhagic stroke of devastating morbidity. Furthermore, QSM is poised to play a key role in measuring ironassociated inflammation in multiple sclerosis, and in overcoming limitations in current gadolinium-dependent MRI protocols. Intravascularly, QSM offers the potential of simple yet robust, noninvasive, challenge-free oxygen consumption measurements, whose further development could significantly impact the imaging strategies of a wide range of diseases, including ischemic stroke and Alzheimer's disease. Finally, QSM has the potential to map mineralization for measuring bone strength, and for monitoring drug biodistribution delivered by nanocarriers containing magnetic cores. In conclusion, we believe that clinicians should consider integrating QSM into their routine imaging practices by including gradient echo sequences with automatic preservation of both phase and magnitude data in all relevant MRI protocols. 


\section{REFERENCES}

1. de Rochefort L, Liu T, Kressler B, et al. Quantitative susceptibility map reconstruction from MR phase data using bayesian regularization: validation and application to brain imaging. Magn Reson Med 2010;63(1):194-206.

2. Wang Y, Liu T. Quantitative susceptibility mapping (QSM): Decoding MRI data for a tissue magnetic biomarker. Magn Reson Med 2015;73(1):82-101.

3. Reichenbach JR, Schweser F, Serres B, Deistung A. Quantitative Susceptibility Mapping: Concepts and Applications. Clin Neuroradiol 2015;25 Suppl 2:225-230.

4. Liu C, Li W, Tong KA, Yeom KW, Kuzminski S. Susceptibility-weighted imaging and quantitative susceptibility mapping in the brain. Journal of magnetic resonance imaging : JMRI 2015;42(1):23-41.

5. Haacke EM, Liu S, Buch S, Zheng W, Wu D, Ye Y. Quantitative susceptibility mapping: current status and future directions. Magnetic resonance imaging 2015;33(1):1-25.

6. Li J, Chang S, Liu T, et al. Reducing the object orientation dependence of susceptibility effects in gradient echo MRI through quantitative susceptibility mapping. Magn Reson Med 2012;68(5):1563-1569.

7. Young IR, Khenia S, Thomas DG, et al. Clinical magnetic susceptibility mapping of the brain. J Comput Assist Tomogr 1987;11(1):2-6.

8. Mahdi K, Wlodarczyk W, Lange T, Scholz R, Jordan A, Wust P. Quantitative mapping of magnetic fluid distributions by means of MR susceptometry. Proc ISMRM Annual Meeting 2001:797.

9. Li L, Leigh JS. Quantifying arbitrary magnetic susceptibility distributions with MR. Magnet Reson Med 2004;51(5):1077-1082.

10. Morgan J, Irarrazaval P. Efficient solving for arbitrary susceptibility distributions using residual difference fields. International Society for Magnetic Resonance in Medicine. Berlin, Germany; 2007. p. 35.

11. Haacke EM, Cheng NY, House MJ, et al. Imaging iron stores in the brain using magnetic resonance imaging. Magnetic resonance imaging 2005;23(1):1-25.

12. Shmueli K, de Zwart JA, van Gelderen P, Li TQ, Dodd SJ, Duyn JH. Magnetic susceptibility mapping of brain tissue in vivo using MRI phase data. Magn Reson Med 2009;62(6):1510-1522.

13. Kressler B, De Rochefort L, Spincemaille P, Liu T, Wang Y. Estimation of Sparse Magnetic Susceptibility Distributions from MRI Using Non-Linear Regularization. Proc ISMRM 2008:1514.

14. Kressler B, de Rochefort L, Liu T, Spincemaille P, Jiang Q, Wang Y. Nonlinear regularization for per voxel estimation of magnetic susceptibility distributions from MRI field maps. IEEE transactions on medical imaging 2010;29(2):273-281.

15. Wang Y, de Rochefort L, Liu T, Kressler B. Magnetic source MRI: a new quantitative imaging of magnetic biomarkers. Conf Proc IEEE Eng Med Biol Soc 2009;2009:53-56.

16. Haacke EM, Tang J, Neelavalli J, Cheng YC. Susceptibility mapping as a means to visualize veins and quantify oxygen saturation. Journal of magnetic resonance imaging : JMRI 2010;32(3):663-676.

17. Liu C. Susceptibility tensor imaging. Magn Reson Med 2010;63(6):1471-1477. 
8. Schweser F, Deistung A, Lehr BW, Reichenbach JR. Differentiation between diamagnetic and paramagnetic cerebral lesions based on magnetic susceptibility mapping. Med Phys 2010;37(10):5165-5178.

9. Wharton S, Bowtell R. Whole-brain susceptibility mapping at high field: a comparison of multiple- and single-orientation methods. NeuroImage 2010;53(2):515-525.

0 . Wharton S, Schafer A, Bowtell R. Susceptibility mapping in the human brain using threshold-based k-space division. Magn Reson Med 2010;63(5):1292-1304.

1. Li W, Wu B, Liu C. Quantitative susceptibility mapping of human brain reflects spatial variation in tissue composition. NeuroImage 2011;55(4):1645-1656.

2. Liu C, Li W, Johnson GA, Wu B. High-field (9.4 T) MRI of brain dysmyelination by quantitative mapping of magnetic susceptibility. NeuroImage 2011;56(3):930-938.

3. Liu T, Khalidov I, de Rochefort L, et al. A novel background field removal method for MRI using projection onto dipole fields (PDF). NMR Biomed 2011;24(9):1129-1136.

4. Liu T, Liu J, de Rochefort L, et al. Morphology enabled dipole inversion (MEDI) from a single-angle acquisition: comparison with COSMOS in human brain imaging. Magnetic resonance in medicine : official journal of the Society of Magnetic Resonance in Medicine / Society of Magnetic Resonance in Medicine 2011;66(3):777-783.

5. Schweser F, Deistung A, Lehr BW, Reichenbach JR. Quantitative imaging of intrinsic magnetic tissue properties using MRI signal phase: an approach to in vivo brain iron metabolism? NeuroImage 2011;54(4):2789-2807.

6. Bilgic B, Pfefferbaum A, Rohlfing T, Sullivan EV, Adalsteinsson E. MRI estimates of brain iron concentration in normal aging using quantitative susceptibility mapping. NeuroImage 2012;59(3):2625-2635.

7. Chen Z, Calhoun V. Computed inverse resonance imaging for magnetic susceptibility map reconstruction. J Comput Assist Tomogr 2012;36(2):265-274.

8. Langkammer C, Schweser F, Krebs N, et al. Quantitative susceptibility mapping (QSM) as a means to measure brain iron? A post mortem validation study. NeuroImage 2012;62(3):1593-1599.

9. Li X, Vikram DS, Lim IA, Jones CK, Farrell JA, van Zijl PC. Mapping magnetic susceptibility anisotropies of white matter in vivo in the human brain at $7 \mathrm{~T}$. NeuroImage 2012;62(1):314-330.

0. Liu J, Liu T, de Rochefort L, et al. Morphology enabled dipole inversion for quantitative susceptibility mapping using structural consistency between the magnitude image and the susceptibility map. NeuroImage 2012;59(3):2560-2568.

1. Liu T, Xu W, Spincemaille P, Avestimehr AS, Wang Y. Accuracy of the Morphology Enabled Dipole Inversion (MEDI) Algorithm for Quantitative Susceptibility Mapping in MRI. IEEE transactions on medical imaging 2012;31(3):816-824.

2. Lotfipour AK, Wharton S, Schwarz ST, et al. High resolution magnetic susceptibility mapping of the substantia nigra in Parkinson's disease. Journal of magnetic resonance imaging : JMRI 2012;35(1):48-55.

3. Schafer A, Forstmann BU, Neumann J, et al. Direct visualization of the subthalamic nucleus and its iron distribution using high-resolution susceptibility mapping. Hum Brain Mapp 2012;33(12):2831-2842.

4. Schweser F, Sommer K, Deistung A, Reichenbach JR. Quantitative susceptibility mapping for investigating subtle susceptibility variations in the human brain. NeuroImage 2012;62(3):2083-2100. 
5. Wong R, Chen X, Wang Y, Hu X, Jin MM. Visualizing and quantifying acute inflammation using ICAM-1 specific nanoparticles and MRI quantitative susceptibility mapping. Annals of biomedical engineering 2012;40(6):1328-1338.

6. Wu B, Li W, Guidon A, Liu C. Whole brain susceptibility mapping using compressed sensing. Magn Reson Med 2012;67(1)(1):137-147.

7. Deh K, Nguyen TD, Eskreis-Winkler S, et al. Reproducibility of quantitative susceptibility mapping in the brain at two field strengths from two vendors. Journal of magnetic resonance imaging : JMRI 2015;42(6):1592-1600.

8. Hinoda T, Fushimi Y, Okada T, et al. Quantitative Susceptibility Mapping at 3 T and 1.5 T: Evaluation of Consistency and Reproducibility. Invest Radiol 2015;50(8):522-530.

9. Lin PY, Chao TC, Wu ML. Quantitative susceptibility mapping of human brain at 3T: a multisite reproducibility study. AJNR American journal of neuroradiology 2015;36(3):467-474.

0 . Santin MD, Didier M, Valabregue R, et al. Reproducibility of R2 * and quantitative susceptibility mapping (QSM) reconstruction methods in the basal ganglia of healthy subjects. NMR Biomed 2016.

1. Wisnieff C, Liu T, Spincemaille P, Wang S, Zhou D, Wang Y. Magnetic susceptibility anisotropy: cylindrical symmetry from macroscopically ordered anisotropic molecules and accuracy of MRI measurements using few orientations. NeuroImage 2013;70:363376.

2. Wang Y. Principles of Magnetic Resonance Imaging: Physics Concepts, Pulse Sequences, \& Biomedical Applications: CreateSpace Independent Publishing Platform: 2012.

3. Bishop CM. Pattern recognition and machine learning. New York: Springer: 2006. xx, 738 p. p.

4. Smith SM. Fast robust automated brain extraction. Hum Brain Mapp 2002;17(3):143-155.

5. Dong J, Liu T, Chen F, et al. Simultaneous phase unwrapping and removal of chemical shift (SPURS) using graph cuts: application in quantitative susceptibility mapping. IEEE transactions on medical imaging 2015;34(2):531-540.

6. Dimov AV, Liu T, Spincemaille P, et al. Joint estimation of chemical shift and quantitative susceptibility mapping (chemical QSM). Magn Reson Med 2015;73(6):21002110.

7. Liu Z, Yao Y, Zhang Y, Wang Y. QSM0 - QSM with automatic uniform CSF zero reference ISMRM. Honolulu; 2017.

8. Haynes WM. CRC Handbook of Chemistry and Physics, 97th Edition: CRC Press: 2016.

9. Coey JMD. Magnetism and Magnetic Materials: Cambridge University Press: 2010.

0. Guyton AC, Hall JE. Textbook of medical physiology. Philadelphia: Elsevier Saunders: 2006. xxxv, 1116 p. p.

1. Iyengar GV. Elemental Analysis of Biological Systems: CRC-Press: 1989.

2. Zumdahl SS, Zumdahl SA. Chemistry: Cengage Learning: 2006.

3. Harrison PM, Arosio P. The ferritins: molecular properties, iron storage function and cellular regulation. Biochim Biophys Acta 1996;1275(3):161-203.

4. Finazzi D, Arosio P. Biology of ferritin in mammals: an update on iron storage, oxidative damage and neurodegeneration. Archives of toxicology 2014;88(10):1787-1802.

5. Sun H, Walsh AJ, Lebel RM, et al. Validation of quantitative susceptibility mapping with Perls' iron staining for subcortical gray matter. NeuroImage 2015;105:486-492. 
6. Plietker B. Iron Catalysis: Fundamentals and Applications: Springer Berlin Heidelberg: 2011.

7. Pauling L, Coryell CD. The Magnetic Properties and Structure of Hemoglobin, Oxyhemoglobin and Carbonmonoxyhemoglobin. Proc Natl Acad Sci U S A 1936;22(4):210-216.

8. Fan AP, Bilgic B, Gagnon L, et al. Quantitative oxygenation venography from MRI phase. Magn Reson Med 2013;10.1002/mrm.24918.

9. Xu B, Liu T, Spincemaille P, Prince M, Wang Y. Flow compensated quantitative susceptibility mapping for venous oxygenation imaging. Magn Reson Med 2014;72(2):438-445.

0. Dlouhy AC, Outten CE. The iron metallome in eukaryotic organisms. Met Ions Life Sci 2013;12:241-278.

1. Hentze MW, Muckenthaler MU, Andrews NC. Balancing acts: molecular control of mammalian iron metabolism. Cell 2004;117(3):285-297.

2. Brittenham GM. Pathophysiology of iron homeostasis. . In: Hoffman R BE, Shattil SJ, Furie B, Silberstein LE, McGlave P, Heslop H. , editor. Hematology: Basic Principles and Practice. 6th ed. New York: Elsevier; 2013. p. 427-436.

3. Deane R, Zheng W, Zlokovic BV. Brain capillary endothelium and choroid plexus epithelium regulate transport of transferrin-bound and free iron into the rat brain. Journal of neurochemistry 2004;88(4):813-820.

4. Belaidi AA, Bush AI. Iron neurochemistry in Alzheimer's disease and Parkinson's disease: targets for therapeutics. Journal of neurochemistry 2016;139 Suppl 1:179-197.

5. De Domenico I, Vaughn MB, Li L, et al. Ferroportin-mediated mobilization of ferritin iron precedes ferritin degradation by the proteasome. EMBO J 2006;25(22):5396-5404.

6. Zucca FA, Segura-Aguilar J, Ferrari E, et al. Interactions of iron, dopamine and neuromelanin pathways in brain aging and Parkinson's disease. Prog Neurobiol 2015.

7. Fischbach FA, Gregory DW, Harrison PM, Hoy TG, Williams JM. On the structure of hemosiderin and its relationship to ferritin. J Ultrastruct Res 1971;37(5):495-503.

8. Miyazaki E, Kato J, Kobune M, et al. Denatured H-ferritin subunit is a major constituent of haemosiderin in the liver of patients with iron overload. Gut 2002;50(3):413-419.

9. Plascencia-Villa G, Ponce A, Collingwood JF, et al. High-resolution analytical imaging and electron holography of magnetite particles in amyloid cores of Alzheimer's disease. Sci Rep 2016;6:24873.

0 . Rouault TA. Iron metabolism in the CNS: implications for neurodegenerative diseases. Nature reviews Neuroscience 2013;14(8):551-564.

1. Kowal SL, Dall TM, Chakrabarti R, Storm MV, Jain A. The current and projected economic burden of Parkinson's disease in the United States. Movement disorders : official journal of the Movement Disorder Society 2013;28(3):311-318.

2. Braak H, Del Tredici K, Rub U, de Vos RA, Jansen Steur EN, Braak E. Staging of brain pathology related to sporadic Parkinson's disease. Neurobiology of aging 2003;24(2):197-211.

3. Parkinson J. An essay on the shaking palsy. 1817. The Journal of neuropsychiatry and clinical neurosciences 2002;14(2):223-236; discussion 222.

4. Ayton S, Lei P. Nigral iron elevation is an invariable feature of Parkinson's disease and is a sufficient cause of neurodegeneration. BioMed research international 2014;2014:581256. 
5. Gotz ME, Double K, Gerlach M, Youdim MB, Riederer P. The relevance of iron in the pathogenesis of Parkinson's disease. Annals of the New York Academy of Sciences 2004;1012:193-208.

6. Zecca L, Gallorini M, Schunemann V, et al. Iron, neuromelanin and ferritin content in the substantia nigra of normal subjects at different ages: consequences for iron storage and neurodegenerative processes. Journal of neurochemistry 2001;76(6):1766-1773.

7. Dexter DT, Wells FR, Lees AJ, et al. Increased nigral iron content and alterations in other metal ions occurring in brain in Parkinson's disease. Journal of neurochemistry 1989;52(6):1830-1836.

8. Martin WR, Wieler M, Gee M. Midbrain iron content in early Parkinson disease: a potential biomarker of disease status. Neurology 2008;70(16 Pt 2):1411-1417.

9. Ryvlin P, Broussolle E, Piollet H, Viallet F, Khalfallah Y, Chazot G. Magnetic resonance imaging evidence of decreased putamenal iron content in idiopathic Parkinson's disease. Archives of neurology 1995;52(6):583-588.

0. Ulla M, Bonny JM, Ouchchane L, Rieu I, Claise B, Durif F. Is R2* a new MRI biomarker for the progression of Parkinson's disease? A longitudinal follow-up. PLoS One 2013;8(3):e57904.

1. Mastroberardino PG, Hoffman EK, Horowitz MP, et al. A novel transferrin/TfR2mediated mitochondrial iron transport system is disrupted in Parkinson's disease. Neurobiology of disease 2009;34(3):417-431.

2. Salazar J, Mena N, Hunot S, et al. Divalent metal transporter 1 (DMT1) contributes to neurodegeneration in animal models of Parkinson's disease. Proc Natl Acad Sci U S A 2008;105(47):18578-18583.

3. Jin L, Wang J, Zhao L, et al. Decreased serum ceruloplasmin levels characteristically aggravate nigral iron deposition in Parkinson's disease. Brain : a journal of neurology 2011;134(Pt 1):50-58.

4. Dexter DT, Carter CJ, Wells FR, et al. Basal lipid peroxidation in substantia nigra is increased in Parkinson's disease. Journal of neurochemistry 1989;52(2):381-389.

5. Sian J, Dexter DT, Lees AJ, et al. Alterations in glutathione levels in Parkinson's disease and other neurodegenerative disorders affecting basal ganglia. Annals of neurology 1994;36(3):348-355.

6. Alam ZI, Jenner A, Daniel SE, et al. Oxidative DNA damage in the parkinsonian brain: an apparent selective increase in 8-hydroxyguanine levels in substantia nigra. Journal of neurochemistry 1997;69(3):1196-1203.

7. Spillantini MG, Schmidt ML, Lee VM, Trojanowski JQ, Jakes R, Goedert M. Alphasynuclein in Lewy bodies. Nature 1997;388(6645):839-840.

8. Lin MT, Beal MF. Mitochondrial dysfunction and oxidative stress in neurodegenerative diseases. Nature 2006;443(7113):787-795.

9. Mehta V, Pei W, Yang G, et al. Iron is a sensitive biomarker for inflammation in multiple sclerosis lesions. PLoS One 2013;8(3):e57573.

0. Pisanu A, Lecca D, Mulas G, et al. Dynamic changes in pro- and anti-inflammatory cytokines in microglia after PPAR-gamma agonist neuroprotective treatment in the MPTPp mouse model of progressive Parkinson's disease. Neurobiology of disease 2014;71:280-291.

1. Powell LW, Seckington RC, Deugnier Y. Haemochromatosis. Lancet 2016;388(10045):706-716. 
2. Brittenham GM, Badman DG. Noninvasive measurement of iron: report of an NIDDK workshop. Blood 2003;101(1):15-19.

3. Deugnier Y, Turlin B. Pathology of hepatic iron overload. Seminars in liver disease 2011;31(3):260-271.

4. Pietrangelo A. Iron and the liver. Liver Int 2016;36 Suppl 1:116-123.

5. Schaible UE, Kaufmann SH. Iron and microbial infection. Nat Rev Microbiol 2004;2(12):946-953.

6. Cairo G, Recalcati S, Mantovani A, Locati M. Iron trafficking and metabolism in macrophages: contribution to the polarized phenotype. Trends Immunol 2011;32(6):241247.

7. Nairz M, Schroll A, Sonnweber T, Weiss G. The struggle for iron - a metal at the hostpathogen interface. Cell Microbiol 2010;12(12):1691-1702.

8. McFarland HF, Martin R. Multiple sclerosis: a complicated picture of autoimmunity. Nature immunology 2007;8(9):913-919.

9. Lassmann H, van Horssen J, Mahad D. Progressive multiple sclerosis: pathology and pathogenesis. Nature reviews Neurology 2012;8(11):647-656.

00. Trapp BD, Nave KA. Multiple sclerosis: an immune or neurodegenerative disorder? Annual review of neuroscience 2008;31:247-269.

01. Bagnato F, Hametner S, Yao B, et al. Tracking iron in multiple sclerosis: a combined imaging and histopathological study at 7 Tesla. Brain : a journal of neurology 2011;134(Pt 12):3602-3615.

02. Wisnieff C, Ramanan S, Olesik J, Gauthier S, Wang Y, Pitt D. Quantitative susceptibility mapping (QSM) of white matter multiple sclerosis lesions: Interpreting positive susceptibility and the presence of iron. Magn Reson Med 2015;74(2):564-570.

03. Hametner S, Wimmer I, Haider L, Pfeifenbring S, Bruck W, Lassmann H. Iron and neurodegeneration in the multiple sclerosis brain. Annals of neurology 2013;74(6):848861.

04. Drayer B, Burger P, Hurwitz B, Dawson D, Cain J. Reduced signal intensity on MR images of thalamus and putamen in multiple sclerosis: increased iron content? AJR Am J Roentgenol 1987;149(2):357-363.

05. Bakshi R, Dmochowski J, Shaikh ZA, Jacobs L. Gray matter T2 hypointensity is related to plaques and atrophy in the brains of multiple sclerosis patients. J Neurol Sci 2001;185(1):19-26.

06. Sun X, He G, Qing H, et al. Hypoxia facilitates Alzheimer's disease pathogenesis by upregulating BACE1 gene expression. Proc Natl Acad Sci U S A 2006;103(49):1872718732.

07. Trapp BD, Stys PK. Virtual hypoxia and chronic necrosis of demyelinated axons in multiple sclerosis. Lancet Neurol 2009;8(3):280-291.

08. Baron JC. Mapping the ischaemic penumbra with PET: implications for acute stroke treatment. Cerebrovasc Dis 1999;9(4):193-201.

09. Robertson CS, Narayan RK, Gokaslan ZL, et al. Cerebral arteriovenous oxygen difference as an estimate of cerebral blood flow in comatose patients. J Neurosurg 1989;70(2):222-230.

10. van Asch CJ, Luitse MJ, Rinkel GJ, van der Tweel I, Algra A, Klijn CJ. Incidence, case fatality, and functional outcome of intracerebral haemorrhage over time, according to age, 
sex, and ethnic origin: a systematic review and meta-analysis. Lancet Neurol 2010;9(2):167-176.

11. Greenberg SM, Vernooij MW, Cordonnier C, et al. Cerebral microbleeds: a guide to detection and interpretation. Lancet Neurol 2009;8(2):165-174.

12. Chang S, Zhang J, Liu T, et al. Quantitative Susceptibility Mapping of Intracerebral Hemorrhages at Various Stages. Journal of magnetic resonance imaging : JMRI 2016;44(2):420-425.

13. Bradley WG, Jr., Schmidt PG. Effect of methemoglobin formation on the MR appearance of subarachnoid hemorrhage. Radiology 1985;156(1):99-103.

14. Xi G, Keep RF, Hoff JT. Mechanisms of brain injury after intracerebral haemorrhage. Lancet Neurol 2006;5(1):53-63.

15. Keep RF, Hua Y, Xi G. Intracerebral haemorrhage: mechanisms of injury and therapeutic targets. Lancet Neurol 2012;11(8):720-731.

16. Proudfoot D, Skepper JN, Hegyi L, Bennett MR, Shanahan CM, Weissberg PL. Apoptosis regulates human vascular calcification in vitro: evidence for initiation of vascular calcification by apoptotic bodies. Circ Res 2000;87(11):1055-1062.

17. Raisz LG. Pathogenesis of osteoporosis: concepts, conflicts, and prospects. J Clin Invest 2005;115(12):3318-3325.

18. Scimeca M, Giannini E, Antonacci C, Pistolese CA, Spagnoli LG, Bonanno E. Microcalcifications in breast cancer: an active phenomenon mediated by epithelial cells with mesenchymal characteristics. BMC Cancer 2014;14:286.

19. Wu B, Pei X, Li ZY. How does calcification influence plaque vulnerability? Insights from fatigue analysis. ScientificWorldJournal 2014;2014:417324.

20. Lohrke J, Frenzel T, Endrikat J, et al. 25 Years of Contrast-Enhanced MRI: Developments, Current Challenges and Future Perspectives. Adv Ther 2016;33(1):1-28.

21. Gao J, Gu H, Xu B. Multifunctional magnetic nanoparticles: design, synthesis, and biomedical applications. Acc Chem Res 2009;42(8):1097-1107.

22. Lauffer RB. Paramagnetic metal complexes as water proton relaxation agents for NMR imaging: theory and design. Chem Rev 1987;87:901-927.

23. Wang Y. Principles of Magnetic Resonance Imaging: Physics Concepts, Pulse Sequences, and Biomedical Applications: CreateSpace Independent Publishing Platform: 2012.

24. Bammer R. MR and CT Perfusion and Pharmacokinetic Imaging: Clinical Applications and Theoretical Principles: LWW: 2016.

25. Haacke EM, Reichenbach JrR. Susceptibility weighted imaging in MRI : basic concepts and clinical applications. Hoboken, N.J.: Wiley-Blackwell: 2011. xvi, 743 p. p.

26. Deyos D, French DSG. Patient profile, indications, efficacy and safety of duodenal levodopa infusion in advanced Parkinson's disease. Movement disorders : official journal of the Movement Disorder Society 2009;24(7):993-1000.

27. Devos D, Moreau C, Devedjian JC, et al. Targeting chelatable iron as a therapeutic modality in Parkinson's disease. Antioxid Redox Signal 2014;21(2):195-210.

28. Ide S, Kakeda S, Ueda I, et al. Internal structures of the globus pallidus in patients with Parkinson's disease: evaluation with quantitative susceptibility mapping (QSM). Eur Radiol 2015;25(3):710-718.

29. Lotfipour AK, Wharton S, Schwarz ST, et al. High resolution magnetic susceptibility mapping of the substantia nigra in Parkinson's disease. J Magn Reson Imaging 2012;35(1):48-55. 
30. Azuma M, Hirai T, Yamada K, et al. Lateral Asymmetry and Spatial Difference of Iron Deposition in the Substantia Nigra of Patients with Parkinson Disease Measured with Quantitative Susceptibility Mapping. AJNR American journal of neuroradiology 2016;37(5):782-788.

31. Barbosa JH, Santos AC, Tumas V, et al. Quantifying brain iron deposition in patients with Parkinson's disease using quantitative susceptibility mapping, R2 and R2. Magnetic resonance imaging 2015;33(5):559-565.

32. Du G, Liu T, Lewis MM, et al. Quantitative susceptibility mapping of the midbrain in Parkinson's disease. Movement disorders : official journal of the Movement Disorder Society 2016;31(3):317-324.

33. Guan X, Xuan M, Gu Q, et al. Regionally progressive accumulation of iron in Parkinson's disease as measured by quantitative susceptibility mapping. NMR Biomed 2016.

34. He N, Ling H, Ding B, et al. Region-specific disturbed iron distribution in early idiopathic Parkinson's disease measured by quantitative susceptibility mapping. Hum Brain Mapp 2015;36(11):4407-4420.

35. Murakami Y, Kakeda S, Watanabe K, et al. Usefulness of quantitative susceptibility mapping for the diagnosis of Parkinson disease. AJNR American journal of neuroradiology 2015;36(6):1102-1108.

36. Acosta-Cabronero J, Cardenas-Blanco A, Betts MJ, et al. The whole-brain pattern of magnetic susceptibility perturbations in Parkinson's disease. Brain : a journal of neurology 2017;140(Pt 1):118-131.

37. Okun MS. Deep-brain stimulation for Parkinson's disease. The New England journal of medicine 2012;367(16):1529-1538.

38. Massey LA, Miranda MA, Zrinzo L, et al. High resolution MR anatomy of the subthalamic nucleus: imaging at $9.4 \mathrm{~T}$ with histological validation. NeuroImage 2012;59(3):2035-2044.

39. Wodarg F, Herzog J, Reese R, et al. Stimulation site within the MRI-defined STN predicts postoperative motor outcome. Movement disorders : official journal of the Movement Disorder Society 2012;27(7):874-879.

40. Liu T, Eskreis-Winkler S, Schweitzer AD, et al. Improved subthalamic nucleus depiction with quantitative susceptibility mapping. Radiology 2013;269(1):216-223.

41. Chandran AS, Bynevelt M, Lind CR. Magnetic resonance imaging of the subthalamic nucleus for deep brain stimulation. J Neurosurg 2016;124(1):96-105.

42. Brittenham GM. Iron-chelating therapy for transfusional iron overload. The New England journal of medicine 2011;364(2):146-156.

43. Poggiali E, Cassinerio E, Zanaboni L, Cappellini MD. An update on iron chelation therapy. Blood Transfus 2012;10(4):411-422.

44. Abbruzzese $\mathrm{G}$, Cossu G, Balocco M, et al. A pilot trial of deferiprone for neurodegeneration with brain iron accumulation. Haematologica 2011;96(11):1708-1711.

45. Langkammer C, Pirpamer L, Seiler S, et al. Quantitative Susceptibility Mapping in Parkinson's Disease. PLoS One 2016;11(9):e0162460.

46. Ward RJ, Dexter DT, Crichton RR. Neurodegenerative diseases and therapeutic strategies using iron chelators. Journal of trace elements in medicine and biology : organ of the Society for Minerals and Trace Elements 2015. 
47. Acosta-Cabronero J, Williams GB, Cardenas-Blanco A, Arnold RJ, Lupson V, Nestor PJ. In vivo quantitative susceptibility mapping (QSM) in Alzheimer's disease. PLoS One 2013;8(11):e81093.

48. Moon Y, Han SH, Moon WJ. Patterns of Brain Iron Accumulation in Vascular Dementia and Alzheimer's Dementia Using Quantitative Susceptibility Mapping Imaging. J Alzheimers Dis 2016;51(3):737-745.

49. Hwang EJ, Kim HG, Kim D, et al. Texture analyses of quantitative susceptibility maps to differentiate Alzheimer's disease from cognitive normal and mild cognitive impairment. Med Phys 2016;43(8):4718.

50. van Bergen JM, Hua J, Unschuld PG, et al. Quantitative Susceptibility Mapping Suggests Altered Brain Iron in Premanifest Huntington Disease. AJNR American journal of neuroradiology 2016;37(5):789-796.

51. Deistung A, Stefanescu MR, Ernst TM, et al. Structural and Functional Magnetic Resonance Imaging of the Cerebellum: Considerations for Assessing Cerebellar Ataxias. Cerebellum 2016;15(1):21-25.

52. Schweitzer AD, Liu T, Gupta A, et al. Quantitative susceptibility mapping of the motor cortex in amyotrophic lateral sclerosis and primary lateral sclerosis. AJR Am J Roentgenol 2015;204(5):1086-1092.

53. Kamm CP, Uitdehaag BM, Polman CH. Multiple Sclerosis: Current Knowledge and Future Outlook. European neurology 2014;72(3-4):132-141.

54. Vellinga MM, Oude Engberink RD, Seewann A, et al. Pluriformity of inflammation in multiple sclerosis shown by ultra-small iron oxide particle enhancement. Brain : a journal of neurology 2008;131(Pt 3):800-807.

55. Wiggermann V, Hernandez Torres E, Vavasour IM, et al. Magnetic resonance frequency shifts during acute MS lesion formation. Neurology 2013;81(3):211-218.

56. Langkammer C, Liu T, Khalil M, et al. Quantitative susceptibility mapping in multiple sclerosis. Radiology 2013;267(2):551-559.

57. Schmalbrock P, Prakash RS, Schirda B, et al. Basal Ganglia Iron in Patients with Multiple Sclerosis Measured with 7T Quantitative Susceptibility Mapping Correlates with Inhibitory Control. AJNR American journal of neuroradiology 2016;37(3):439-446.

58. Bian W, Tranvinh E, Tourdias T, et al. In Vivo 7T MR Quantitative Susceptibility Mapping Reveals Opposite Susceptibility Contrast between Cortical and White Matter Lesions in Multiple Sclerosis. AJNR American journal of neuroradiology 2016.

59. Kakeda S, Futatsuya K, Ide S, et al. Improved Detection of Cortical Gray Matter Involvement in Multiple Sclerosis with Quantitative Susceptibility Mapping. Acad Radiol 2015;22(11):1427-1432.

60. Cobzas D, Sun H, Walsh AJ, Lebel RM, Blevins G, Wilman AH. Subcortical gray matter segmentation and voxel-based analysis using transverse relaxation and quantitative susceptibility mapping with application to multiple sclerosis. Journal of magnetic resonance imaging : JMRI 2015;42(6):1601-1610.

61. Chen W, Gauthier SA, Gupta A, et al. Quantitative susceptibility mapping of multiple sclerosis lesions at various ages. Radiology 2014;271(1):183-192.

62. Cronin MJ, Wharton S, Al-Radaideh A, et al. A comparison of phase imaging and quantitative susceptibility mapping in the imaging of multiple sclerosis lesions at ultrahigh field. MAGMA 2016;29(3):543-557. 
63. Eskreis-Winkler S, Deh K, Gupta A, et al. Multiple sclerosis lesion geometry in quantitative susceptibility mapping (QSM) and phase imaging. Journal of magnetic resonance imaging: JMRI 2015;42(1):224-229.

64. Harrison DM, Li X, Liu H, et al. Lesion Heterogeneity on High-Field Susceptibility MRI Is Associated with Multiple Sclerosis Severity. AJNR American journal of neuroradiology 2016;37(8):1447-1453.

65. Li X, Harrison DM, Liu H, et al. Magnetic susceptibility contrast variations in multiple sclerosis lesions. Journal of magnetic resonance imaging : JMRI 2016;43(2):463-473.

66. Zhang Y, Gauthier SA, Gupta A, et al. Quantitative Susceptibility Mapping and R2* Measured Changes during White Matter Lesion Development in Multiple Sclerosis:

Myelin Breakdown, Myelin Debris Degradation and Removal, and Iron Accumulation. AJNR American journal of neuroradiology 2016;37(9):1629-1635.

67. Zhang Y, Gauthier SA, Gupta A, et al. Longitudinal change in magnetic susceptibility of new enhanced multiple sclerosis (MS) lesions measured on serial quantitative susceptibility mapping (QSM). Journal of magnetic resonance imaging : JMRI 2016;44(2):426-432.

68. Zhang Y, Gauthier SA, Gupta A, et al. Magnetic Susceptibility from Quantitative Susceptibility Mapping Can Differentiate New Enhancing from Nonenhancing Multiple Sclerosis Lesions without Gadolinium Injection. AJNR American journal of neuroradiology 2016.

69. Absinta M, Sati P, Schindler M, et al. Persistent 7-tesla phase rim predicts poor outcome in new multiple sclerosis patient lesions. J Clin Invest 2016;126(7):2597-2609.

70. Kolb-Sobieraj C, Gupta S, Weinstock-Guttman B. Laquinimod therapy in multiple sclerosis: a comprehensive review. Neurol Ther 2014;3(1):29-39.

71. Kappos L, Li DK, Stuve O, et al. Safety and Efficacy of Siponimod (BAF312) in Patients With Relapsing-Remitting Multiple Sclerosis: Dose-Blinded, Randomized Extension of the Phase 2 BOLD Study. JAMA Neurol 2016;73(9):1089-1098.

72. Durafourt BA, Lambert C, Johnson TA, Blain M, Bar-Or A, Antel JP. Differential responses of human microglia and blood-derived myeloid cells to FTY720. J Neuroimmunol 2011;230(1-2):10-16.

73. Mishra MK, Wang J, Keough MB, et al. Laquinimod reduces neuroaxonal injury through inhibiting microglial activation. Ann Clin Transl Neurol 2014;1(6):409-422.

74. Kanda T, Osawa M, Oba H, et al. High Signal Intensity in Dentate Nucleus on Unenhanced T1-weighted MR Images: Association with Linear versus Macrocyclic Gadolinium Chelate Administration. Radiology 2015;275(3):803-809.

75. Radbruch A, Weberling LD, Kieslich PJ, et al. Gadolinium retention in the dentate nucleus and globus pallidus is dependent on the class of contrast agent. Radiology 2015;275(3):783-791.

76. Roccatagliata L, Vuolo L, Bonzano L, Pichiecchio A, Mancardi GL. Multiple sclerosis: hyperintense dentate nucleus on unenhanced T1-weighted MR images is associated with the secondary progressive subtype. Radiology 2009;251(2):503-510.

77. Shinohara RT, Goldsmith J, Mateen FJ, Crainiceanu C, Reich DS. Predicting breakdown of the blood-brain barrier in multiple sclerosis without contrast agents. AJNR American journal of neuroradiology 2012;33(8):1586-1590. 
78. Blystad I, Hakansson I, Tisell A, et al. Quantitative MRI for Analysis of Active Multiple Sclerosis Lesions without Gadolinium-Based Contrast Agent. AJNR American journal of neuroradiology 2015.

79. Lo CP, Kao HW, Chen SY, et al. Comparison of diffusion-weighted imaging and contrast-enhanced T1-weighted imaging on a single baseline MRI for demonstrating dissemination in time in multiple sclerosis. BMC Neurol 2014;14:100.

80. Sati P, Oh J, Constable RT, et al. The central vein sign and its clinical evaluation for the diagnosis of multiple sclerosis: a consensus statement from the North American Imaging in Multiple Sclerosis Cooperative. Nature reviews Neurology 2016;12(12):714-722.

81. Ogasawara A, Kakeda S, Watanabe K, et al. Quantitative susceptibility mapping in patients with systemic lupus erythematosus: detection of abnormalities in normalappearing basal ganglia. Eur Radiol 2016;26(4):1056-1063.

82. Hernando D, Levin YS, Sirlin CB, Reeder SB. Quantification of liver iron with MRI: state of the art and remaining challenges. Journal of magnetic resonance imaging : JMRI 2014;40(5):1003-1021.

83. Sharma P, Altbach M, Galons JP, Kalb B, Martin DR. Measurement of liver fat fraction and iron with MRI and MR spectroscopy techniques. Diagn Interv Radiol 2014;20(1):1726.

84. Wood JC. Estimating tissue iron burden: current status and future prospects. British journal of haematology 2015;170(1):15-28.

85. Quinn CT, St Pierre TG. MRI Measurements of Iron Load in Transfusion-Dependent Patients: Implementation, Challenges, and Pitfalls. Pediatr Blood Cancer 2016;63(5):773780.

86. Yablonskiy DA, Haacke EM. Theory of NMR signal behavior in magnetically inhomogeneous tissues: the static dephasing regime. Magn Reson Med 1994;32(6):749763.

87. Sharma SD, Fischer R, Schoennagel BP, et al. MRI-based quantitative susceptibility mapping (QSM) and R2* mapping of liver iron overload: Comparison with SQUIDbased biomagnetic liver susceptometry. Magn Reson Med 2016.

88. Carpenter KL, Li W, Wei H, et al. Magnetic susceptibility of brain iron is associated with childhood spatial IQ. NeuroImage 2016;132:167-174.

89. Li X, Allen RP, Earley CJ, et al. Brain iron deficiency in idiopathic restless legs syndrome measured by quantitative magnetic susceptibility at 7 tesla. Sleep Med 2016;22:75-82.

90. Jones TH, Morawetz RB, Crowell RM, et al. Thresholds of focal cerebral ischemia in awake monkeys. J Neurosurg 1981;54(6):773-782.

91. Hacke W, Furlan AJ, Al-Rawi Y, et al. Intravenous desmoteplase in patients with acute ischaemic stroke selected by MRI perfusion-diffusion weighted imaging or perfusion CT (DIAS-2): a prospective, randomised, double-blind, placebo-controlled study. Lancet Neurol 2009;8(2):141-150.

92. Young AR, Sette G, Touzani O, et al. Relationships between high oxygen extraction fraction in the acute stage and final infarction in reversible middle cerebral artery occlusion: an investigation in anesthetized baboons with positron emission tomography. $\mathrm{J}$ Cereb Blood Flow Metab 1996;16(6):1176-1188.

93. Lee DH, Kang DW, Ahn JS, Choi CG, Kim SJ, Suh DC. Imaging of the ischemic penumbra in acute stroke. Korean J Radiol 2005;6(2):64-74. 
94. Heiss WD, Huber M, Fink GR, et al. Progressive derangement of periinfarct viable tissue in ischemic stroke. J Cereb Blood Flow Metab 1992;12(2):193-203.

95. Furlan M, Marchal G, Viader F, Derlon JM, Baron JC. Spontaneous neurological recovery after stroke and the fate of the ischemic penumbra. Annals of neurology 1996;40(2):216-226.

96. Wise RJ, Bernardi S, Frackowiak RS, Legg NJ, Jones T. Serial observations on the pathophysiology of acute stroke. The transition from ischaemia to infarction as reflected in regional oxygen extraction. Brain : a journal of neurology 1983;106 (Pt 1):197-222.

97. Raichle ME, Martin WR, Herscovitch P, Mintun MA, Markham J. Brain blood flow measured with intravenous H2(15)O. II. Implementation and validation. J Nucl Med 1983;24(9):790-798.

98. Bolar DS, Rosen BR, Sorensen AG, Adalsteinsson E. QUantitative Imaging of eXtraction of oxygen and TIssue consumption (QUIXOTIC) using venular-targeted velocityselective spin labeling. Magn Reson Med 2011;66(6):1550-1562.

99. Guo J, Wong EC. Venous oxygenation mapping using velocity-selective excitation and arterial nulling. Magn Reson Med 2012;68(5):1458-1471.

00. An H, Lin W. Cerebral venous and arterial blood volumes can be estimated separately in humans using magnetic resonance imaging. Magn Reson Med 2002;48(4):583-588.

01. He X, Yablonskiy DA. Quantitative BOLD: mapping of human cerebral deoxygenated blood volume and oxygen extraction fraction: default state. Magn Reson Med 2007;57(1):115-126.

02. Davis TL, Kwong KK, Weisskoff RM, Rosen BR. Calibrated functional MRI: mapping the dynamics of oxidative metabolism. Proc Natl Acad Sci U S A 1998;95(4):1834-1839.

03. Bulte DP, Kelly M, Germuska M, et al. Quantitative measurement of cerebral physiology using respiratory-calibrated MRI. NeuroImage 2012;60(1):582-591.

04. Gauthier CJ, Hoge RD. Magnetic resonance imaging of resting OEF and CMRO(2) using a generalized calibration model for hypercapnia and hyperoxia. NeuroImage 2012;60(2):1212-1225.

05. Zhang J, Liu T, Gupta A, Spincemaille P, Nguyen TD, Wang Y. Quantitative mapping of cerebral metabolic rate of oxygen (CMRO2 ) using quantitative susceptibility mapping (QSM). Magn Reson Med 2015;74(4):945-952.

06. Zhang J, Zhou D, Nguyen TD, Spincemaille P, Gupta A, Wang Y. Cerebral metabolic rate of oxygen (CMRO2 ) mapping with hyperventilation challenge using quantitative susceptibility mapping (QSM). Magn Reson Med 2016.

07. Zhang J, Zhou D, Eskreis-Winkler S, et al. Quantitative Susceptibility Mapping (QSM) Based Cerebral Metabolic Rate of Oxygen (CMRO2) Mapping: Eliminating Blood Flow Challenge with Minimal Local Variance (MLV). Proc ISMRM Annual Meeting. Singapore; 2016. p. 603.

08. Liu T, Wisnieff C, Lou M, Chen W, Spincemaille P, Wang Y. Nonlinear formulation of the magnetic field to source relationship for robust quantitative susceptibility mapping. Magn Reson Med 2013;69(2):467-476.

09. Liu Z, Kee Y, Zhou D, Wang Y, Spincemaille P. Preconditioned total field inversion (TFI) method for quantitative susceptibility mapping. Magn Reson Med 2016.

10. Sun H, Kate M, Gioia LC, Emery DJ, Butcher K, Wilman AH. Quantitative susceptibility mapping using a superposed dipole inversion method: Application to intracranial hemorrhage. Magn Reson Med 2016;76(3):781-791. 
11. Wei H, Dibb R, Zhou Y, et al. Streaking artifact reduction for quantitative susceptibility mapping of sources with large dynamic range. NMR Biomed 2015;28(10):1294-1303.

12. Wang S, Lou M, Liu T, Cui D, Chen X, Wang Y. Hematoma volume measurement in gradient echo MRI using quantitative susceptibility mapping. Stroke 2013;44(8):23152317.

13. Chen W, Zhu W, Kovanlikaya I, et al. Intracranial calcifications and hemorrhages: characterization with quantitative susceptibility mapping. Radiology 2014;270(2):496505 .

14. Deistung A, Schweser F, Wiestler B, et al. Quantitative susceptibility mapping differentiates between blood depositions and calcifications in patients with glioblastoma. PLoS One 2013;8(3):e57924.

15. Tan H, Zhang L, Mikati AG, et al. Quantitative Susceptibility Mapping in Cerebral

Cavernous Malformations: Clinical Correlations. AJNR American journal of neuroradiology 2016;37(7):1209-1215.

16. Tuhrim S. Intracerebral hemorrhage--improving outcome by reducing volume? The New England journal of medicine 2008;358(20):2174-2176.

17. Selim M, Yeatts S, Goldstein JN, et al. Safety and tolerability of deferoxamine mesylate in patients with acute intracerebral hemorrhage. Stroke 2011;42(11):3067-3074.

18. Liu T, Surapaneni K, Lou M, Cheng L, Spincemaille P, Wang Y. Cerebral microbleeds: burden assessment by using quantitative susceptibility mapping. Radiology 2012;262(1):269-278.

19. Lee SH, Ryu WS, Roh JK. Cerebral microbleeds are a risk factor for warfarin-related intracerebral hemorrhage. Neurology 2009;72(2):171-176.

20. Liu W, Soderlund K, Senseney JS, et al. Imaging Cerebral Microhemorrhages in Military Service Members with Chronic Traumatic Brain Injury. Radiology 2016;278(2):536-545.

21. Wen Y, Nguyen TD, Liu Z, et al. In Vivo Quantitative Susceptibility Mapping (QSM) in Cardiac MRI. Proc ISMRM Annual Meeting. Singapore; 2016. p. 417.

22. Goldfarb JW, Hasan U, Zhao W, Han J. Magnetic resonance susceptibility weighted phase imaging for the assessment of reperfusion intramyocardial hemorrhage. Magn Reson Med 2014;71(3):1210-1220.

23. Betgem RP, de Waard GA, Nijveldt R, Beek AM, Escaned J, van Royen N. Intramyocardial haemorrhage after acute myocardial infarction. Nat Rev Cardiol 2015;12(3):156-167.

24. Tella SH, Gallagher JC. Prevention and treatment of postmenopausal osteoporosis. J Steroid Biochem Mol Biol 2014;142:155-170.

25. Link TM. Radiology of Osteoporosis. Can Assoc Radiol J 2016;67(1):28-40.

26. Black DM, Greenspan SL, Ensrud KE, et al. The effects of parathyroid hormone and alendronate alone or in combination in postmenopausal osteoporosis. The New England journal of medicine 2003;349(13):1207-1215.

27. Dimov A, Liu Z, Spincemaille, P., Wang Y. Bone Quantitative Susceptibility Mapping Using Tissue Specific R2* and Multi-Peak Fat Spectrum to Model Ultra-Short TE Gradient Echo Signal. ISMRM. Singapore; 2016. p. 363.

28. Straub S, Laun FB, Emmerich J, et al. Potential of quantitative susceptibility mapping for detection of prostatic calcifications. Journal of magnetic resonance imaging : JMRI 2016.

29. Michel JB, Virmani R, Arbustini E, Pasterkamp G. Intraplaque haemorrhages as the trigger of plaque vulnerability. Eur Heart J 2011;32(16):1977-1985, 1985a, 1985b, 1985c. 
30. de Rochefort L, Brown R, Prince MR, Wang Y. Quantitative MR susceptibility mapping using piece-wise constant regularized inversion of the magnetic field. Magn Reson Med 2008;60(4):1003-1009.

31. de Rochefort L, Nguyen T, Brown R, et al. In vivo quantification of contrast agent concentration using the induced magnetic field for time-resolved arterial input function measurement with MRI. Med Phys 2008;35(12):5328-5339.

32. Terreno E, Geninatti Crich S, Belfiore S, et al. Effect of the intracellular localization of a $\mathrm{Gd}$-based imaging probe on the relaxation enhancement of water protons. Magn Reson Med 2006;55(3):491-497.

33. Xu B, Spincemaille P, Liu T, et al. Quantification of cerebral perfusion using dynamic quantitative susceptibility mapping. Magn Reson Med 2015;73(4):1540-1548.

34. Bonekamp D, Barker PB, Leigh R, van Zijl PC, Li X. Susceptibility-based analysis of dynamic gadolinium bolus perfusion MRI. Magn Reson Med 2015;73(2):544-554.
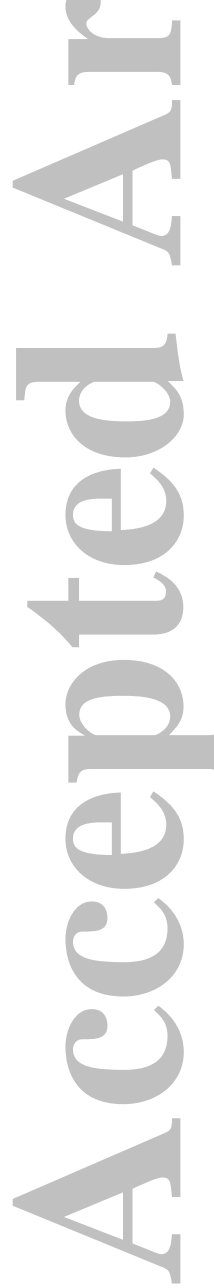


\section{FIGURE LEGENDS}

Figure 1. GRE MRI of a healthy subject: a) magnitude image of $\mathrm{T} 2 *$ weighting $(\mathrm{T} 2 * \mathrm{w}$, $\mathrm{TE}=18 \mathrm{msec}$ ), showing marginal tissue contrast in the basal ganglia gray matter, $\mathrm{b}$ ) phase-derived field image after unwrapping and background field removal, showing substantial contrast within the basal ganglia, c) R2* and d) QSM, showing bright contrast for iron in the globus pallidus (horizontal arrows) and vein (vertical arrows). However, calcification in the ventricle (oblique arrows in c\&d) is shown bright on R2* but dark (negative susceptibility) on QSM.

Figure 2. a) Systemic iron homeostasis. The liver regulates the level of plasma transferrin-bound iron by secreting hepcidin to control iron-recycling by macrophages in the liver and spleen, and to control iron-uptake from the diet by the duodenum. The concentration of blood plasma iron is in equilibrium with the concentrations of iron in all cells through circulation of the labile iron pool. Iron circulation in the body is indicated by blue arrows. Iron can only be absorbed into the body in the duodenum (red arrow), and the body does not have a mechanism to excrete iron except through cell loss. b) Brain iron homeostasis. The labile iron concentration is in equilibrium with the iron contents in all cells (neurons, microglia, oligodendrocytes, and astrocytes). Iron not participating in neurochemistry is stored in ferritin. Iron can be sequestered into the brain from the capillary blood, but there is no known mechanism for iron to be excreted from the brain.

Figure 3. Iron overload in neurodegeneration. Iron can promote the formation of reactive oxygen species and associated oxidative stress. Consequent neurotoxicity includes protein misfolding and damage to mitochondria and other cellular components. When combining with $\alpha$-synuclein, 
iron may accelerate its misfolding. Iron may also contribute to neurodegeneration by causing activation of pro-inflammatory microglia.

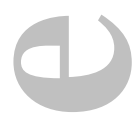

Figure 4. Iron in inflammation. The expression of ferritin and ferroportin in macrophages depend on macrophage activation. a) The classical pro-inflammatory activated (M1) macrophage has high ferritin and low ferroportin, as it tries to limit iron availability to suspected pathogens by sequestering iron from the microenvironment and storing iron. b) On the other extreme, the alternatively activated (M2) macrophage has low ferritin and high ferroportin, as it tries to recirculate iron to the microenvironment for tissue repair.

Figure 5. Calcium homeostasis. Bone is the main site of calcium storage. The concentration of blood plasma calcium is in equilibrium with calcium in all cells through the circulation of labile calcium; this balance is tightly regulated by the thyroid and parathyroid. When plasma calcium is high, the thyroid gland releases calcitonin to stimulate calcium deposition in bones and reduce calcium uptake in the kidneys. When plasma calcium is low, the parathyroid gland secretes parathyroid hormone to stimulate calcium release from bones and increase calcium uptake at the kidneys and intestines.

Figure 6. Pre-surgical mapping for deep brain stimulation (DBS). a) An electrode is inserted into the suthalamic nucleus (STN) during DBS. b) Deep gray nuclei are depicted on QSM, including the globus pallidus externa and interna (GPe, Gpi), substantial nigra (SN), and STN. 
Figure 7. Iron chelation therapy for neurodegenerative diseases. The bidentate ligand deferiprone (DFP) can permeate through the blood brain barrier (BBB). DFP scavenges labile iron that is loosely bound to proteins, forming the 3:1 complex 3DFP+Fe. The complex carries zero charge and diffuses through the $\mathrm{BBB}$, leading to excretion via urine.

Figure 8. Deep gray nuclei depictions on various MRI methods. a) T2 weighted image (T2w), b) $\mathrm{T} 2 *$ weighted image $(\mathrm{T} 2 * \mathrm{~W}, \mathrm{TE}=18 \mathrm{msec})$ or gradient echo magnitude image, $\mathrm{c}) \mathrm{R} 2 *$ mapping, $\mathrm{d})$ phase image with high pass filter, e) susceptibility weighted imaging (SWI), and f) QSM. The nuclei (STN, SN, and GP) are depicted with the best contrast-to-noise ratio on QSM (f). (From https://www.ncbi.nlm.nih.gov/pubmed/23674786)

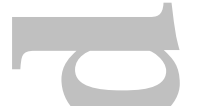

Figure 9. Multiple sclerosis white matter lesion (WM) with a rim of iron and M1 microglia. a) T2 weighted image (T2w) and b) QSM of an MS brain block containing a WM lesion, and of the insert in b, corresponding c) laser ablation inductively coupled plasma mass spectroscopy (LAICP) and d) immunohistochemistry against CD68. Compared to T2w (a), QSM (b) showed a greater volume with a bright rim, which can be biophysically interpreted as containing iron. The rim iron was confirmed on LA-ICP (c) and corresponded to microglia activation (d). (From https://www.ncbi.nlm.nih.gov/pubmed/25137340)

Figure 10a. Iron rim on in vivo QSM. a) T2 weighted image (T2w), b) QSM and c) T1 weighted image with gadolinium injection $(\mathrm{T} 1 \mathrm{w}+\mathrm{Gd})$ of a relapse remitting MS patient. Hyperintense rim on QSM that can be biophysically interpreted as iron is seen on Gd non-enhancing lesions 
(arrows in $\mathrm{a} \& \mathrm{~b}$ ), indicating active chronic inflammation. A QSM isointense lesion is Gd enhancing (circles), suggesting the QSM value is anti-correlated to Gd enhancement.

Figure 10b. QSM allows accurate assessment of MS lesion enhancement status without Gd injection. QSM isointense predicts Gd-enhancing and QSM hyperintense predicts Gd nonenhancing with an area under the receiver operating characteristic curve of 0.96. (From https://www.ncbi.nlm.nih.gov/pubmed/27365331)

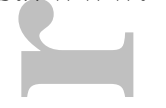

Figure 10c. QSM is ideal to depict the central veins in MS lesions (arrows), while T2w cannot depict the central veins, and $\mathrm{T} 2 * \mathrm{w}$ cannot or only very poorly depict the lesions.

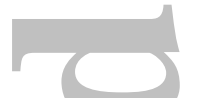

Figure 11. QSM liver iron content quantification. a) Water, b) fat, c) QSM and d) R2* of an axial section of the liver with suspected fibrosis in the medial anterior lobe. Fibrosis did not affect iron quantification by QSM (a) but did affect iron quantification by R2*. QSM overcomes R2* confounding factors, including fibrosis, fat, and edema.

Figure 12. Cerebral metabolic rate of oxygen consumption (CMRO2). a) QSM and b) cerebral blood flow (CBF) were acquired before caffeine challenge, c) QSM and d) CBF after, and e) corresponding CMRO2. QSM is depicted with a gray scale bar $[-50,50] p p b$, CBF with a color scale $\operatorname{bar}[0,150] \mathrm{ml} / 100 \mathrm{~g} / \mathrm{min}$, and QSM with a color scale bar $[0,500] \mu \mathrm{mol} / 100 \mathrm{~g} / \mathrm{min}$. 
Figure 13. Bone QSM with negative susceptibility depicted as bright. The mineralization in the cortical bone of the femur (arrows) is well captured on QSM as depicted on a a) coronal section, b) sagittal section, and c) axial section.

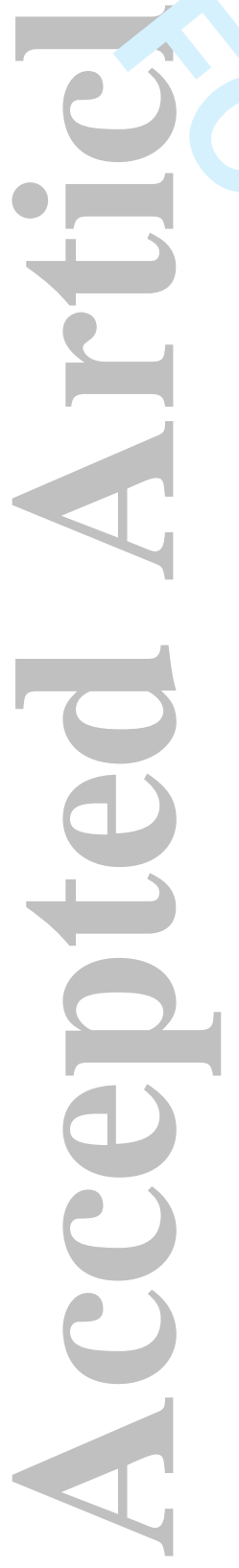

Journal of Magnetic Resonance Imaging 


\section{Figure 1}

a

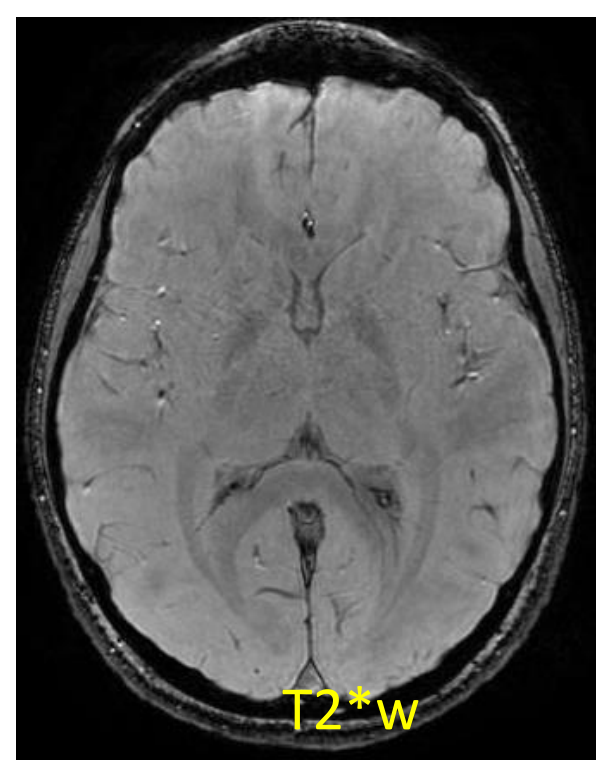

b

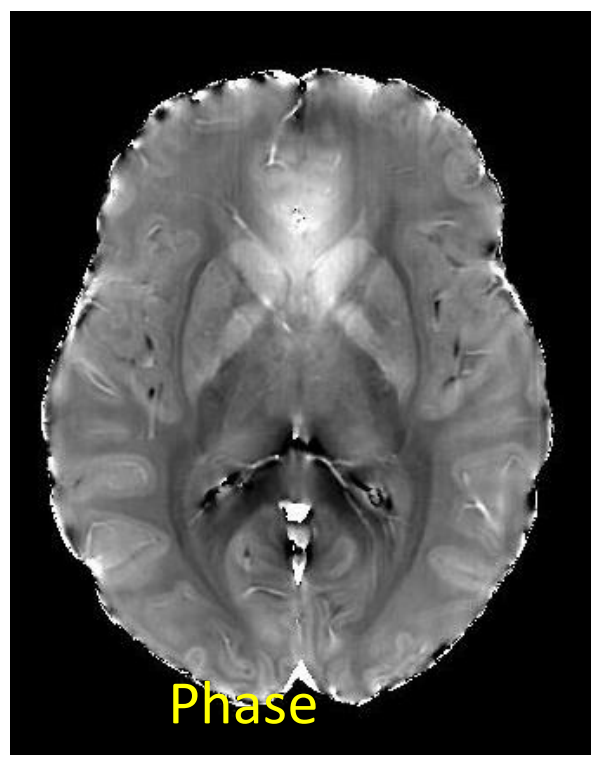

c

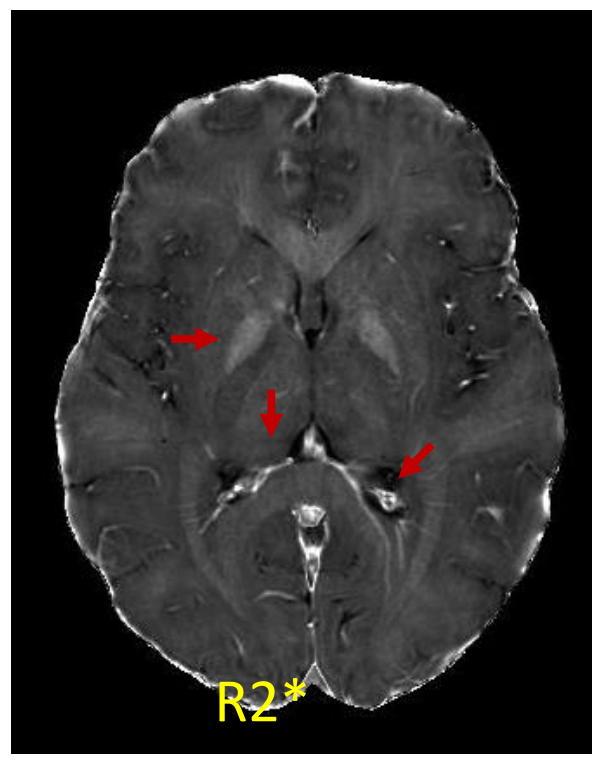

d

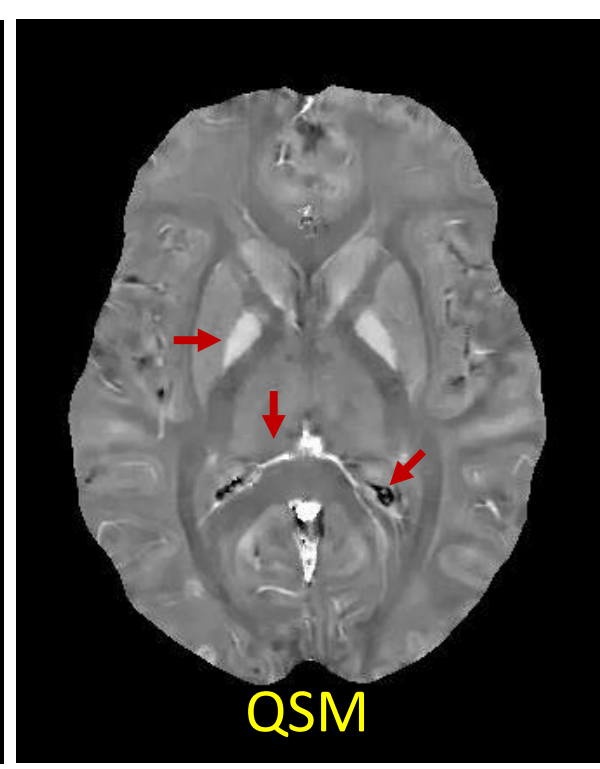

Figure 1. GRE MRI of a healthy subject: a) magnitude image of $\mathrm{T} 2 *$ weighting ( $\mathrm{T} 2 * \mathrm{w}, \mathrm{TE}=18 \mathrm{msec})$, showing marginal tissue contrast in the basal ganglia gray matter, b) phase-derived field image after unwrapping and background field removal, showing substantial contrast within the basal ganglia, c) R2* and d) QSM, showing bright contrast for iron in the globus pallidus (horizontal arrows) and vein (vertical arrows). However, calcification in the ventricle (oblique arrows in c\&d) is shown bright on $\mathrm{R} 2 *$ but dark (negative susceptibility) on QSM. 


\section{Figure $2 a$}

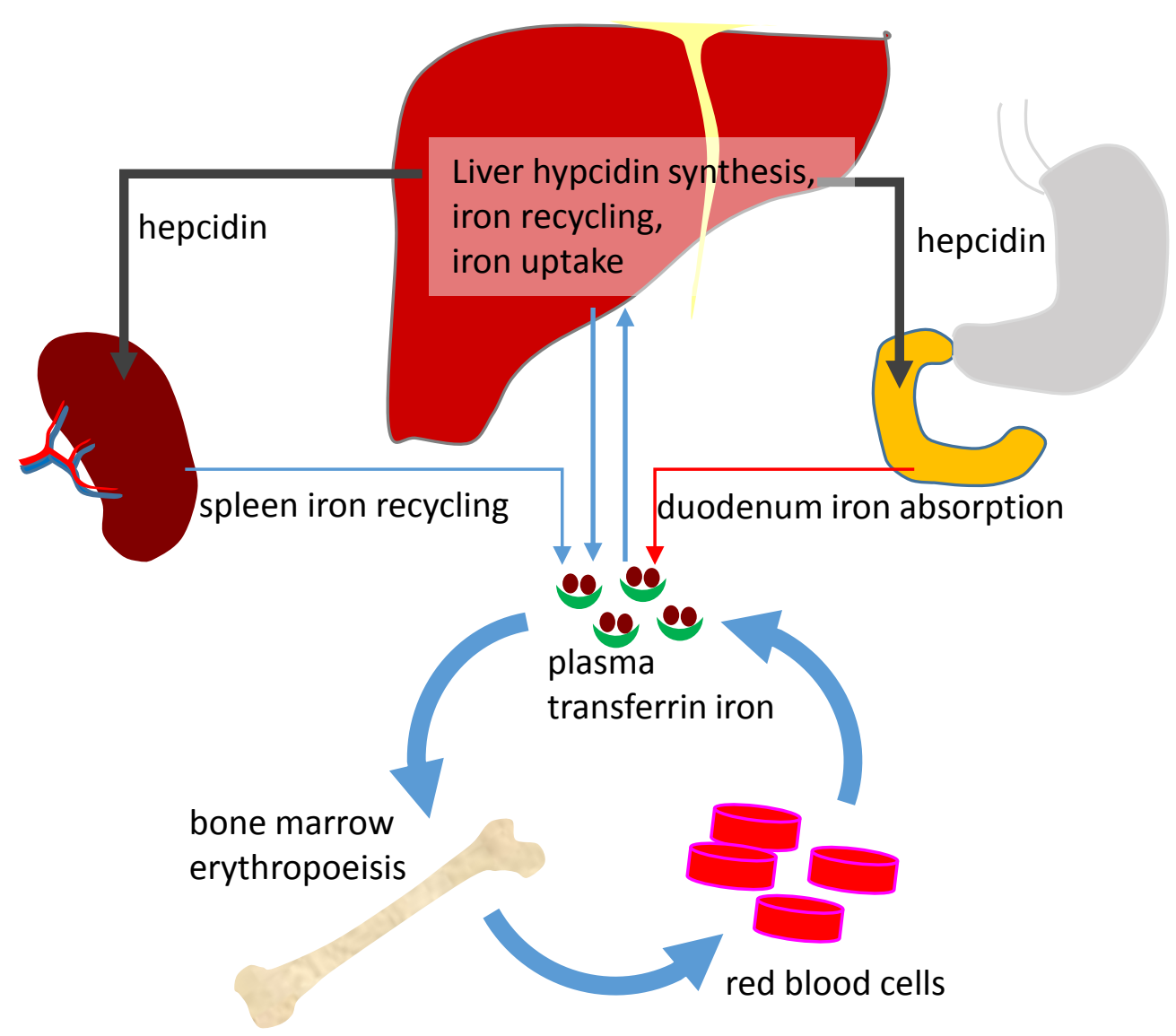

Figure 2. a) Systemic iron homeostasis. The liver regulates the level of plasma transferrin-bound iron by secreting hepcidin to control iron-recycling by macrophages in the liver and spleen, and to control iron-uptake from the diet by the duodenum. The concentration of blood plasma iron is in equilibrium with the concentrations of iron in all cells through circulation of the labile iron pool. Iron circulation in the body is indicated by blue arrows. Iron can only be absorbed into the body in the duodenum (red arrow), and the body does not have a mechanism to excrete iron except through cell loss.

Journal of Magnetic Resonance Imaging

This article is protected by copyright. All rights reserved. 


\section{Figure $2 b$}

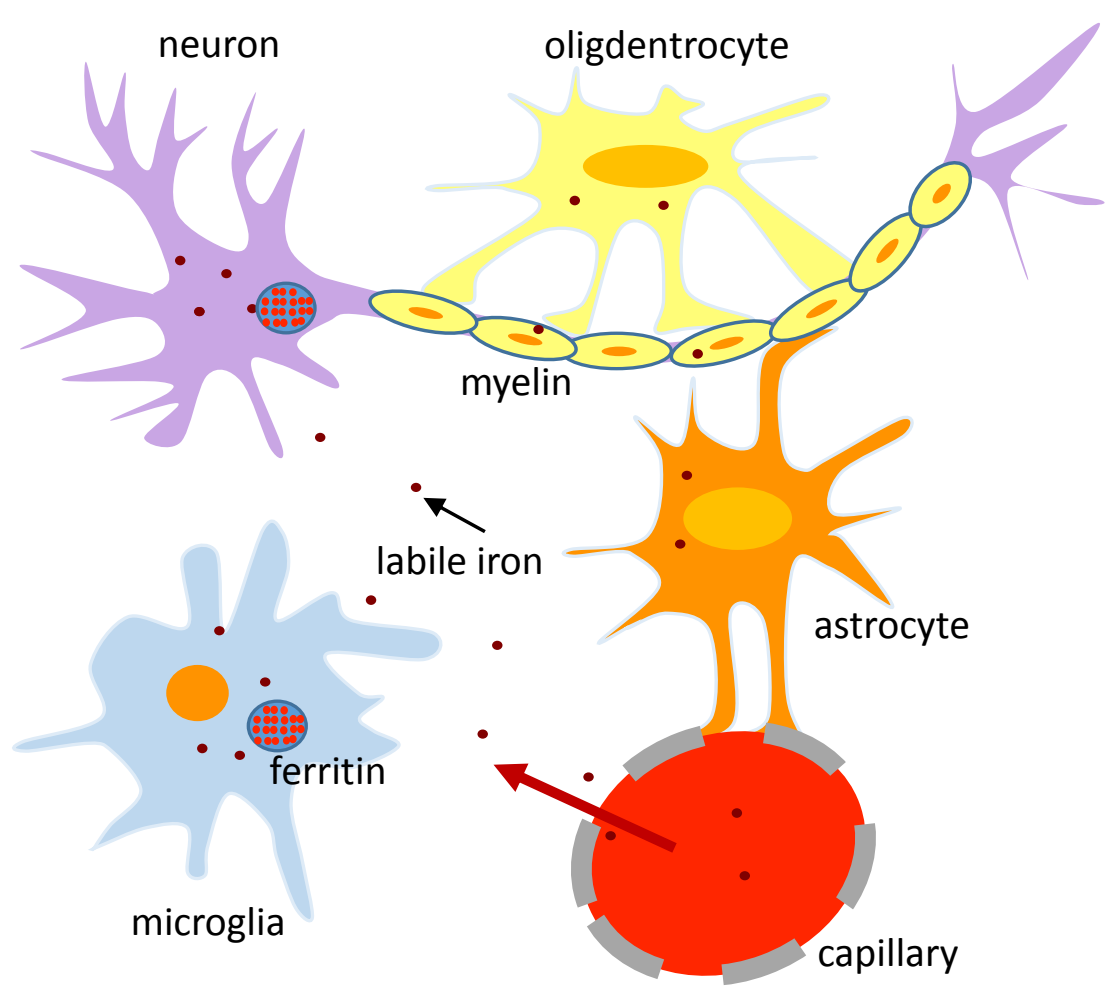

Figure 2. b) Brain iron homeostasis. The labile iron concentration is in equilibrium with the iron contents in all cells (neurons, microglia, oligodendrocytes, and astrocytes). Iron not participating in neurochemistry is stored in ferritin. Iron can be sequestered into the brain from the capillary blood, but there is no known mechanism for iron to be excreted from the brain. 


\section{Figure 3}

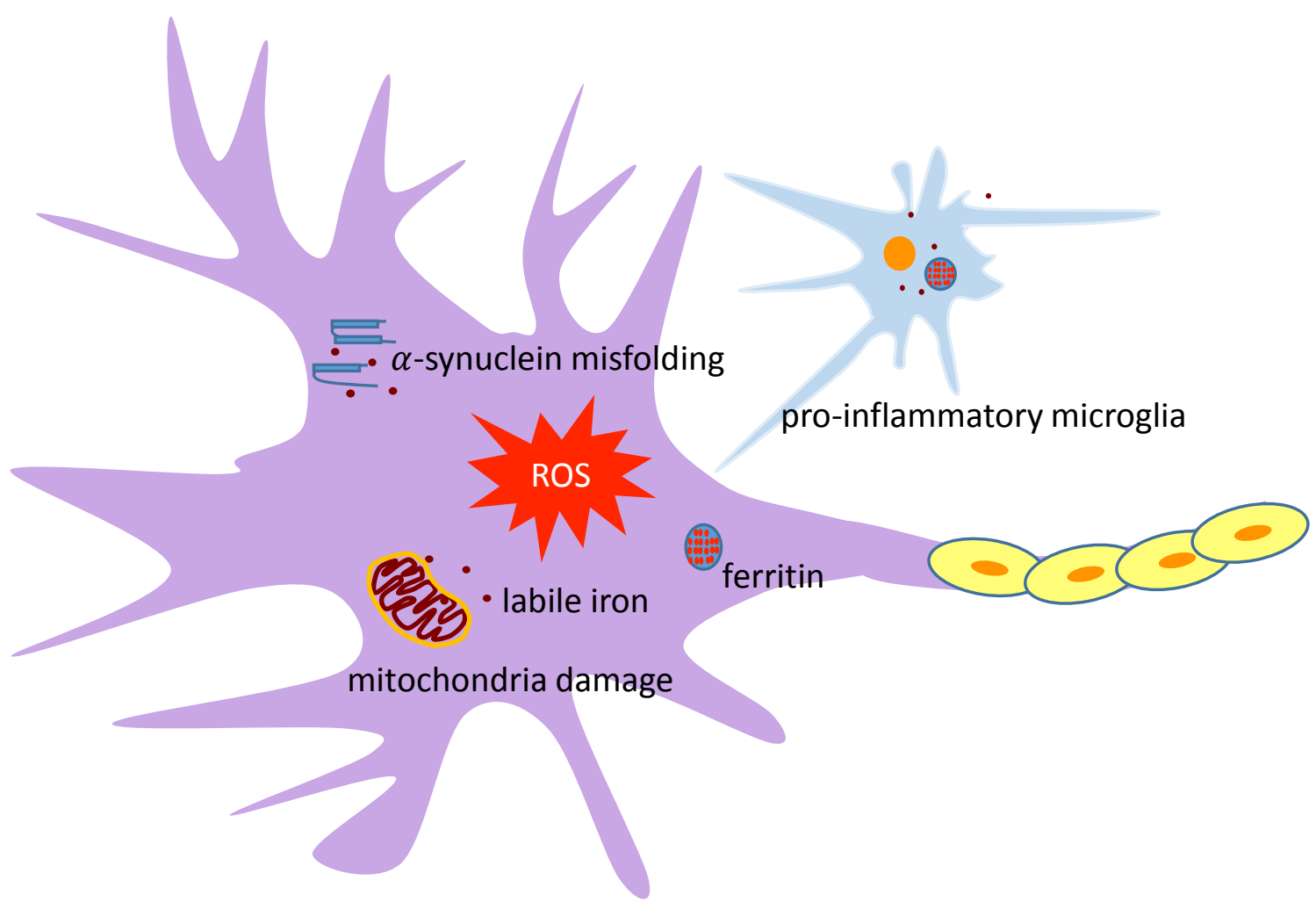

Figure 3. Iron overload in neurodegeneration. Iron can promote the formation of reactive oxygen species and associated oxidative stress. Consequent neurotoxicity includes protein misfolding and damage to mitochondria and other cellular components. When combining with $\alpha$-synuclein, iron may accelerate its misfolding. Iron may also contribute to neurodegeneration by causing activation of pro-inflammatory microglia.

This article is protected by copyright. All rights reserved. 


\section{Figure 4}

$\mathrm{a}$

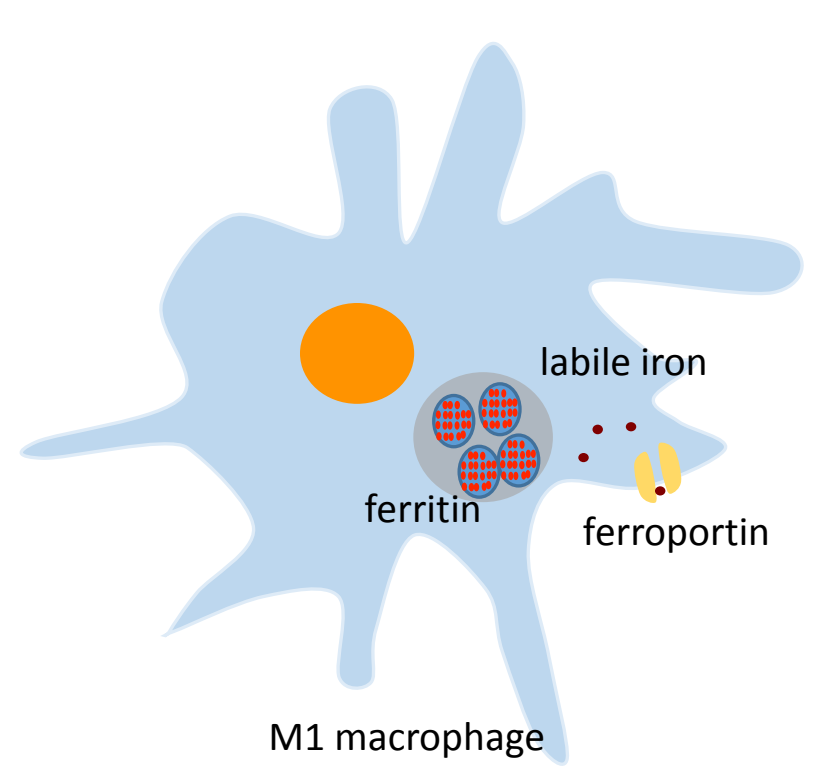

$\mathrm{b}$

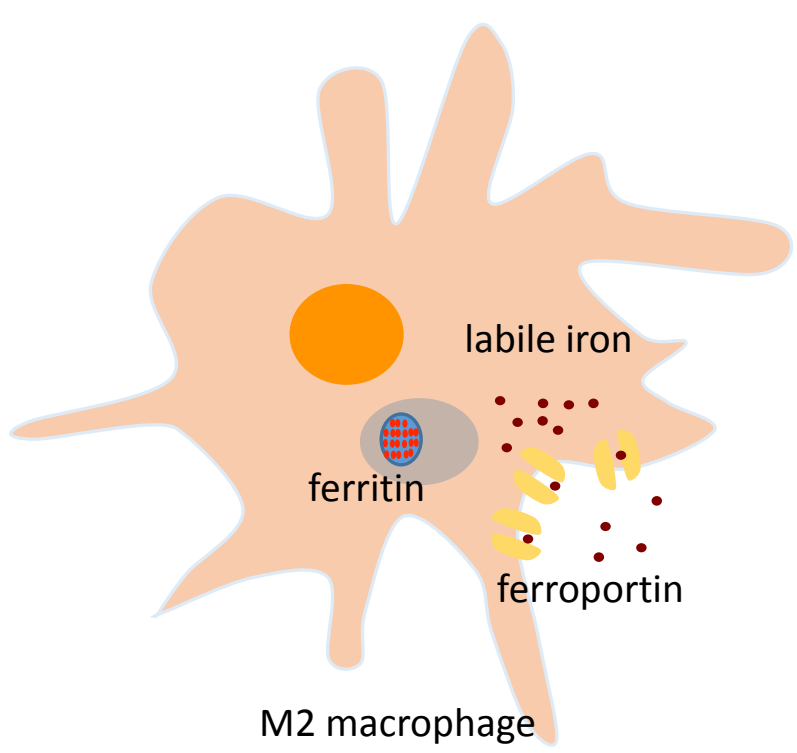

Figure 4. Iron in inflammation. The expression of ferritin and ferroportin in macrophages depend on macrophage activation. a) The classical pro-inflammatory activated (M1) macrophage has high ferritin and low ferroportin, as it tries to limit iron availability to suspected pathogens by sequestering iron from the microenvironment and storing iron. b) On the other extreme, the alternatively activatedo(M2) magrophage has low ferritin and high ferroportin, as it tries to recirculate iron to the microenvironment for tissue repair.

This article is protected by copyright. All rights reserved. 


\section{Figure 5}

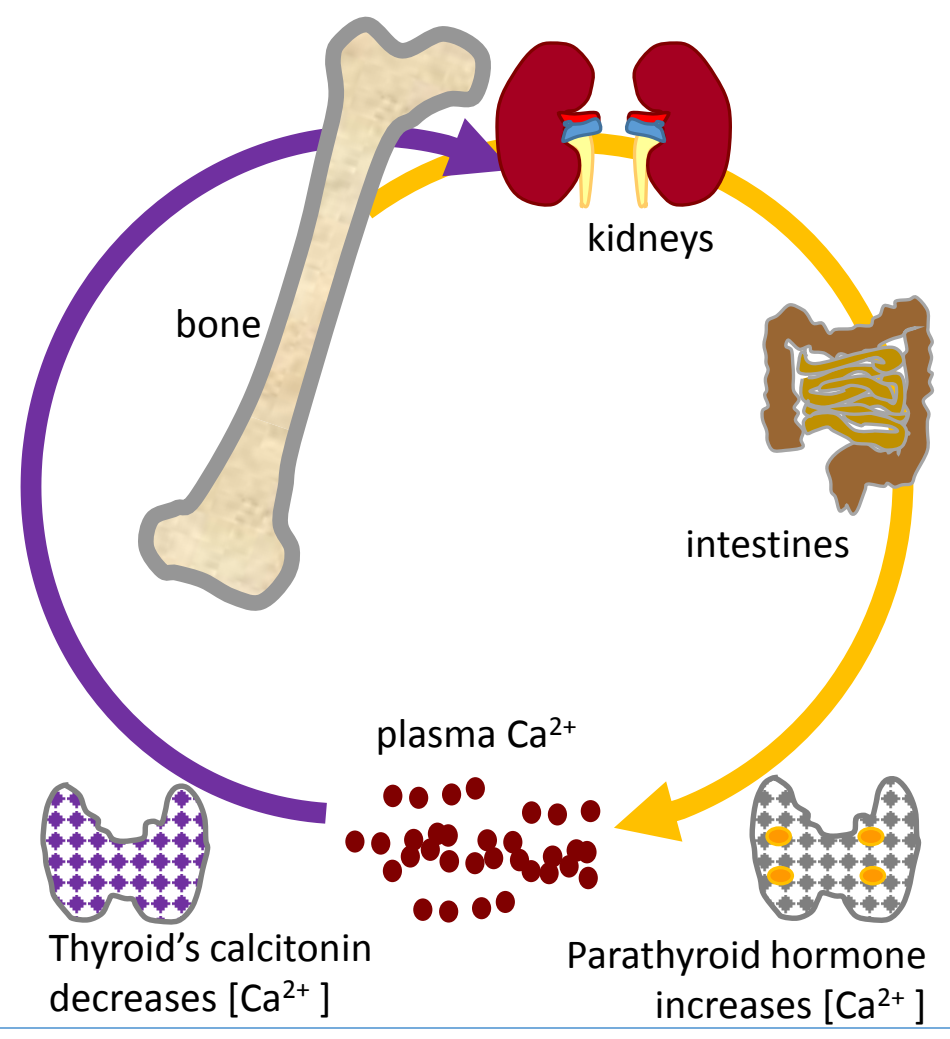

Figure 5. Calcium homeostasis. Bone is the main site of calcium storage. The concentration of blood plasma calcium is in equilibrium with calcium in all cells through the circulation of labile calcium; this balance is tightly regulated by the thyroid and parathyroid. When plasma calcium is high, the thyroid gland releases calcitonin to stimulate calcium deposition in bones and reduce calcium uptake in the kidneys. When plasma calcium is low, the parathyroid gland secretes parathyroid hormone to stimulate calcium release from bones and increase calcium uptake at the kidneys and intestines. 


\section{Figure 6}

a

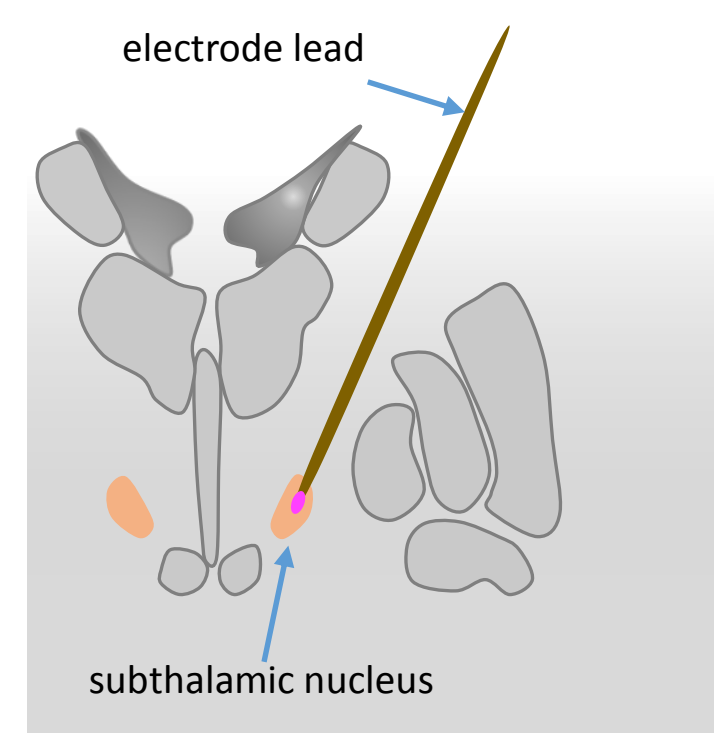

b

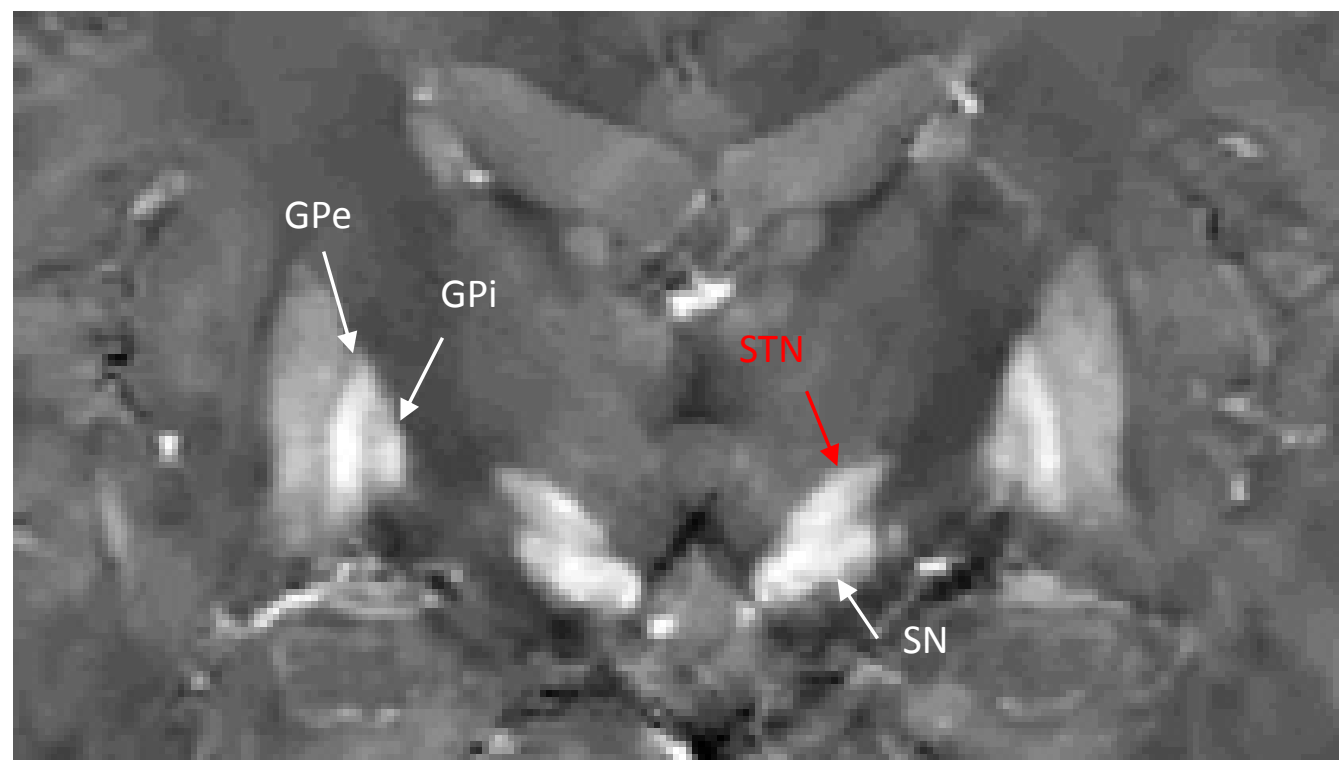

Figure 6. Pre-surgical mapping for deep brain stimulation (DBS). a) An electrode is inserted into the suthalamic nucleus (STN) during DBS. b) Deep gray nuclei are depicted on QSM, including the globus pallidus externa and interna (GPe, Gpi), substantial nigra (SN), and STN. 


\section{Figure 7}

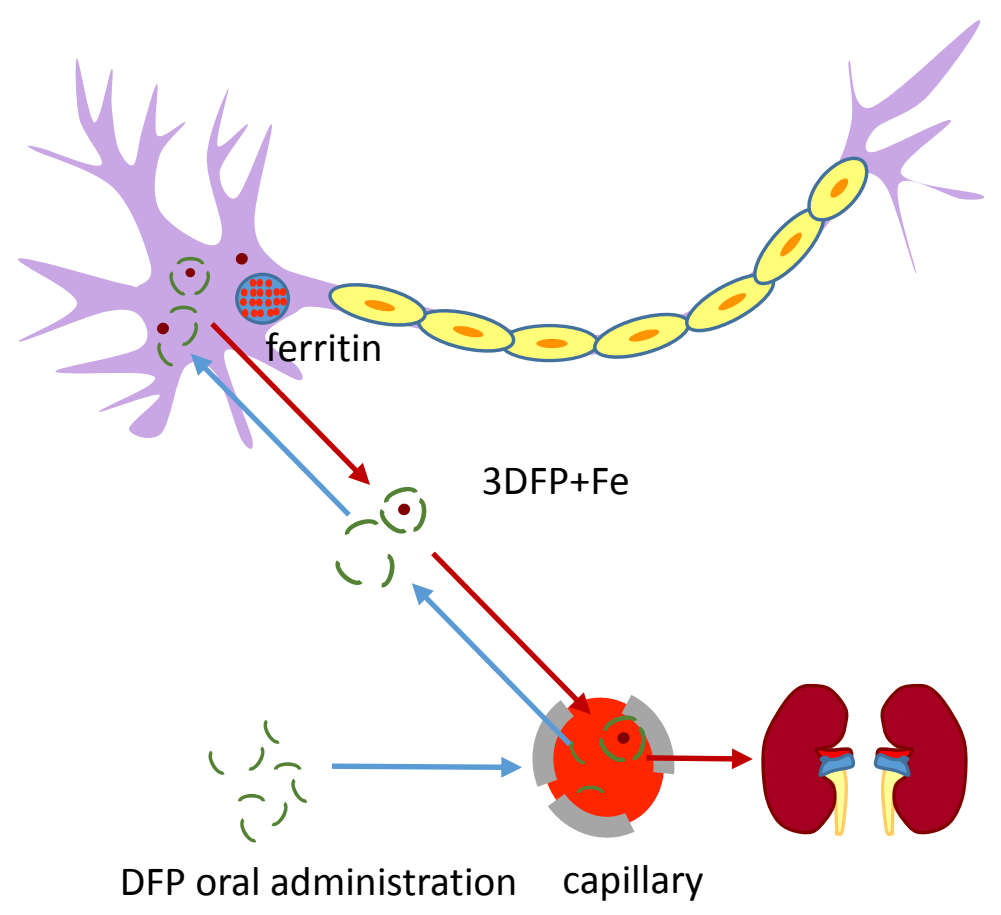

Figure 7. Iron chelation therapy for neurodegenerative diseases. The bidentate ligand deferiprone (DFP) can permeate through the blood brain barrier (BBB). DFP scavenges labile iron that is loosely bound to proteins, forming the 3:1 complex $3 \mathrm{DFP}+\mathrm{Fe}$. The complex carries zero charge and diffuses through the $\mathrm{BBB}$, leading to excretion via urine. 


\section{Figure 8}

$\mathrm{a}$

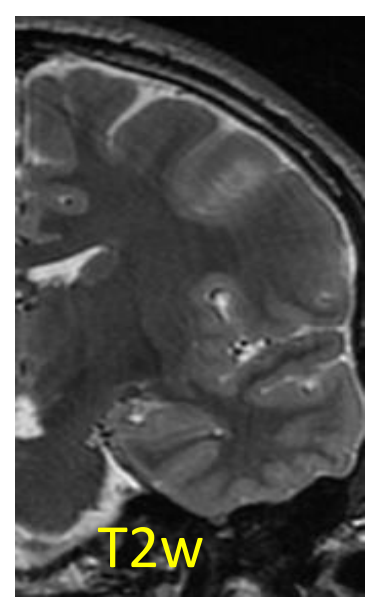

b

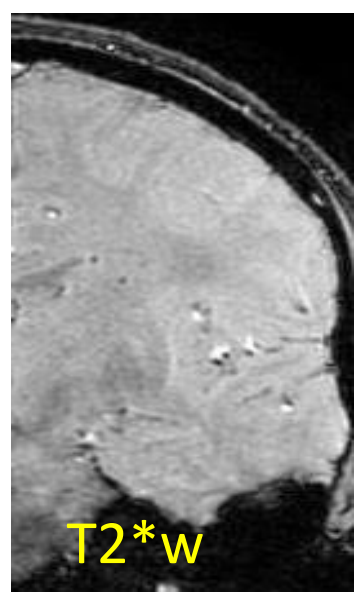

$\mathrm{c}$

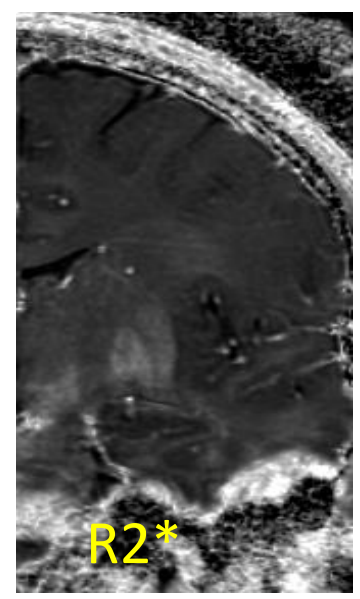

d

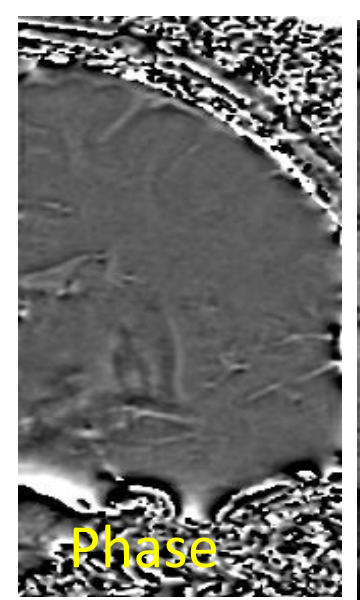

e

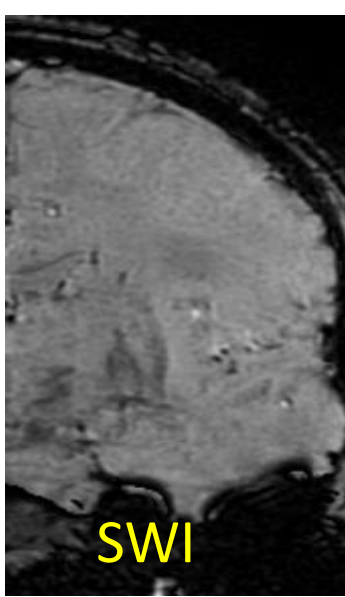

f

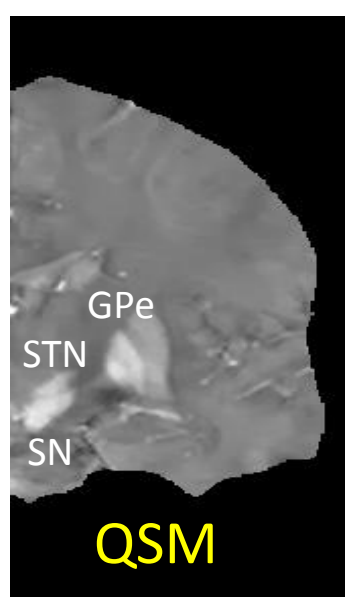

Figure 8. Deep gray nuclei depictions on various MRI methods. a) T2 weighted image (T2w), b) T2* weighted image $(\mathrm{T} 2 * \mathrm{w}, \mathrm{TE}=18 \mathrm{msec})$ or gradient echo magnitude image, c) R2* mapping, d) phase image with high pass filter, e) susceptibility weighted imaging (SWI), and f) QSM. The nuclei (STN, SN, and GP) are depicted with the best contrast-tonoise ratio on QSM (f). (From https://www.ncbi.nlm.nih.gov/pubmed/23674786) 


\section{Figure 9}

a

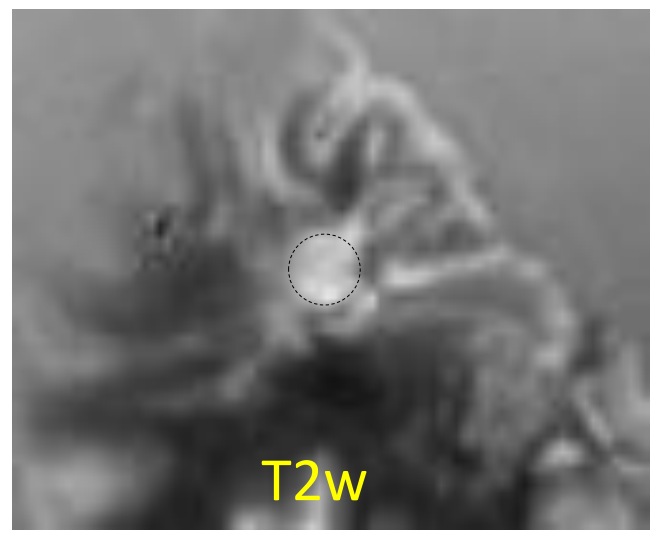

C

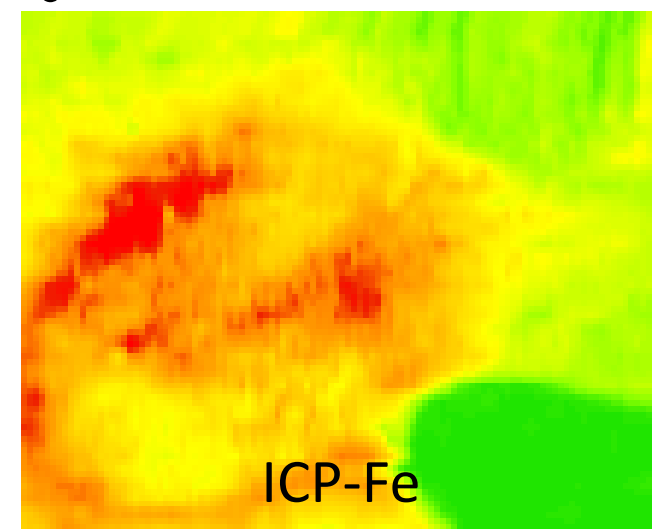

b

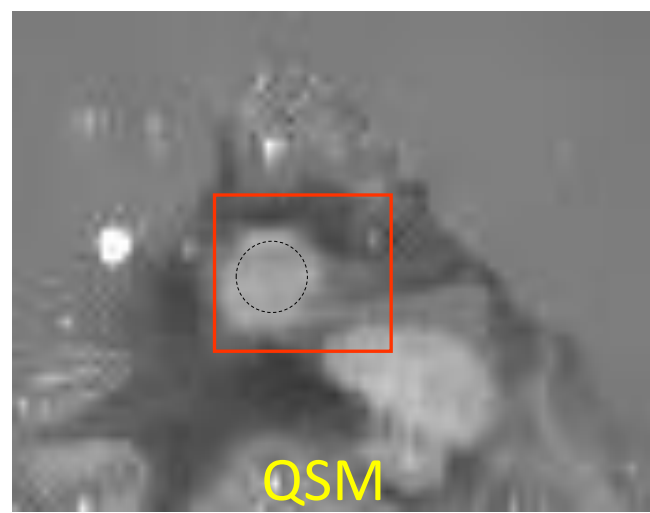

d

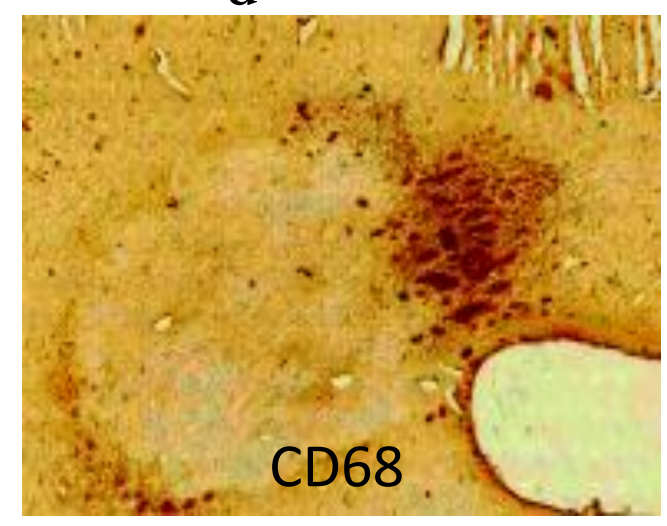

Figure 9. Multiple sclerosis white matter lesion (WM) with a rim of iron and M1 microglia. a) T2 weighted image (T2w) and b) QSM of an MS brain block containing a WM lesion, and of the insert in b, corresponding c) laser ablation inductively coupled plasma mass spectroscopy (LA-ICP) and d) immunohistochemistry against CD68. Compared to T2w (a), QSM (b) showed a greater volume with a bright rim, which can be biophysically interpreted as

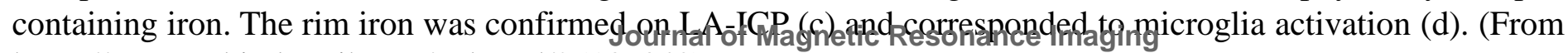
https://www.ncbi.nlm.nih.gov/pubmed/25137340) 


\section{Figure 10a}

a

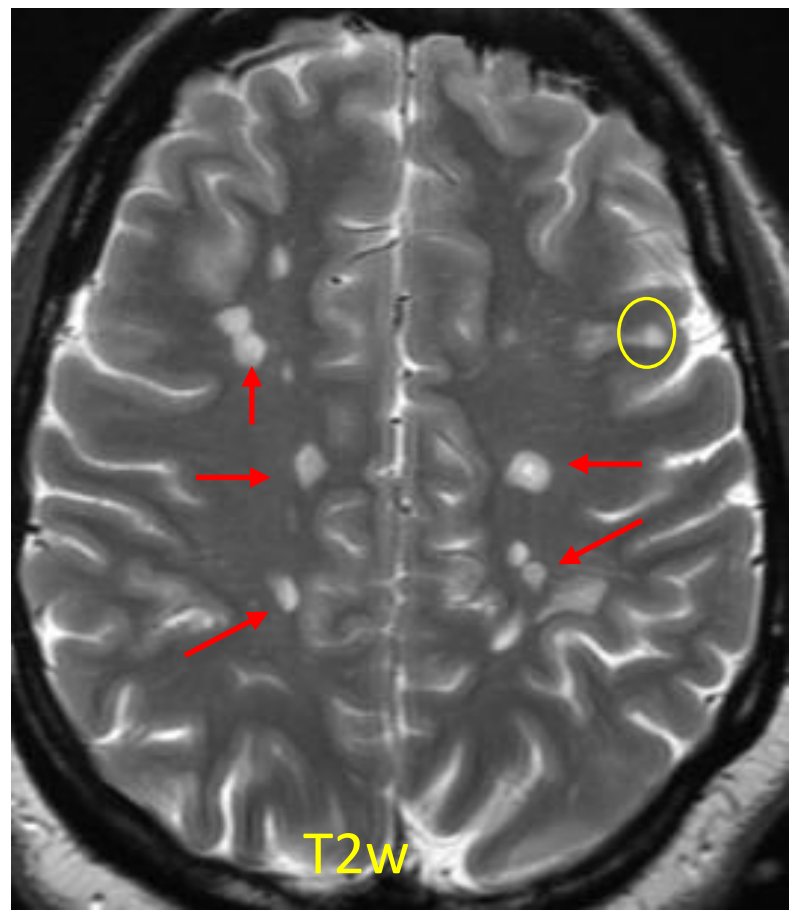

$\mathrm{b}$

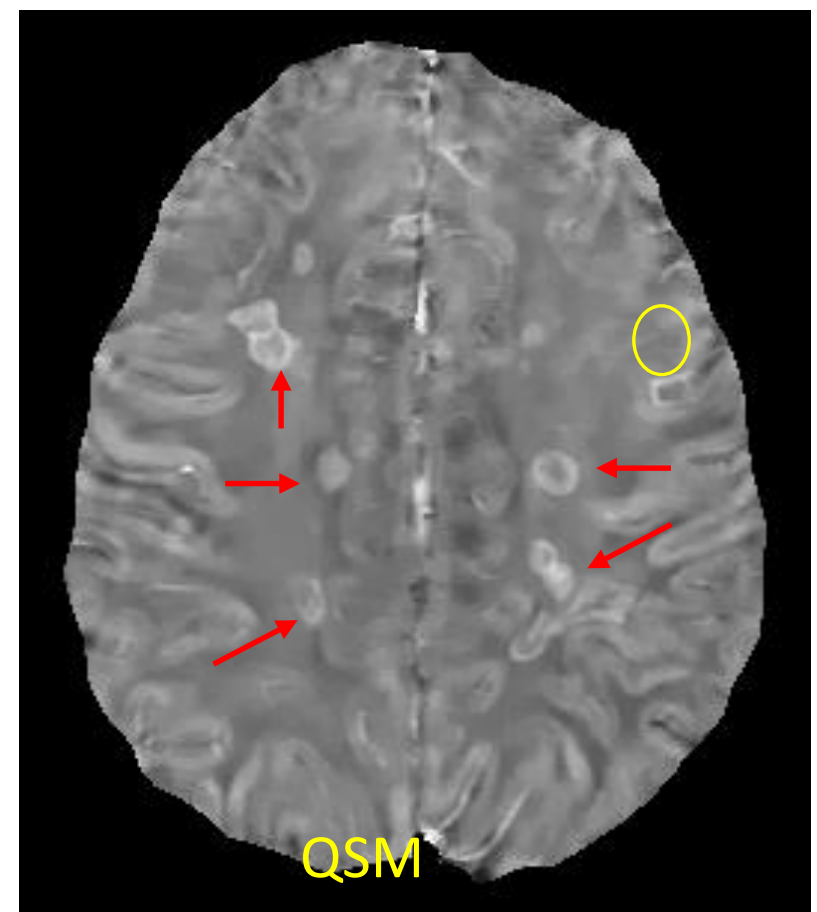

$\mathrm{c}$

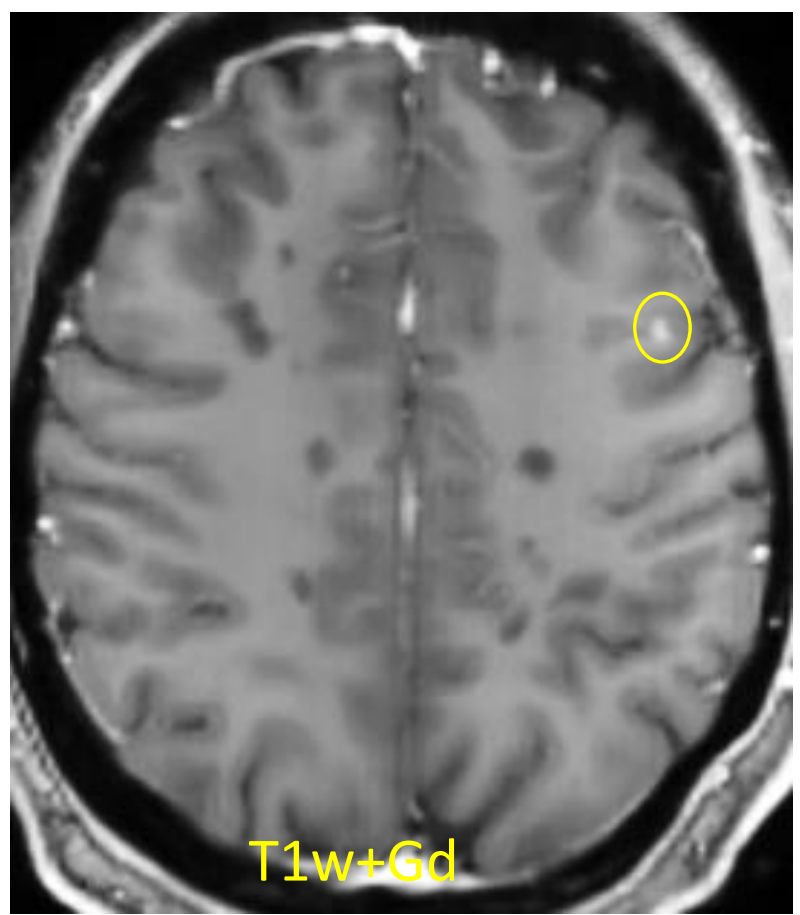

Figure 10. Iron rim on in vivo QSM. a) T2 weighted image (T2w), b) QSM and c) T1 weighted image with gadolinium injection $(\mathrm{T} 1 \mathrm{w}+\mathrm{Gd})$ of a relapse remitting MS patient. Hyperintense rim on QSM that can be biophysically interpreted as

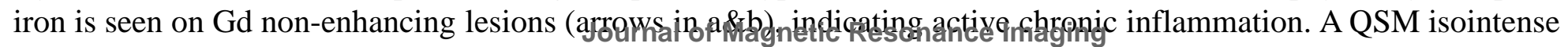
lesion is $\mathrm{Gd}$ enhancing (circles), suggesting the QSM value is anti-correlated to Gd enhancement. 


\section{Figure 10b}

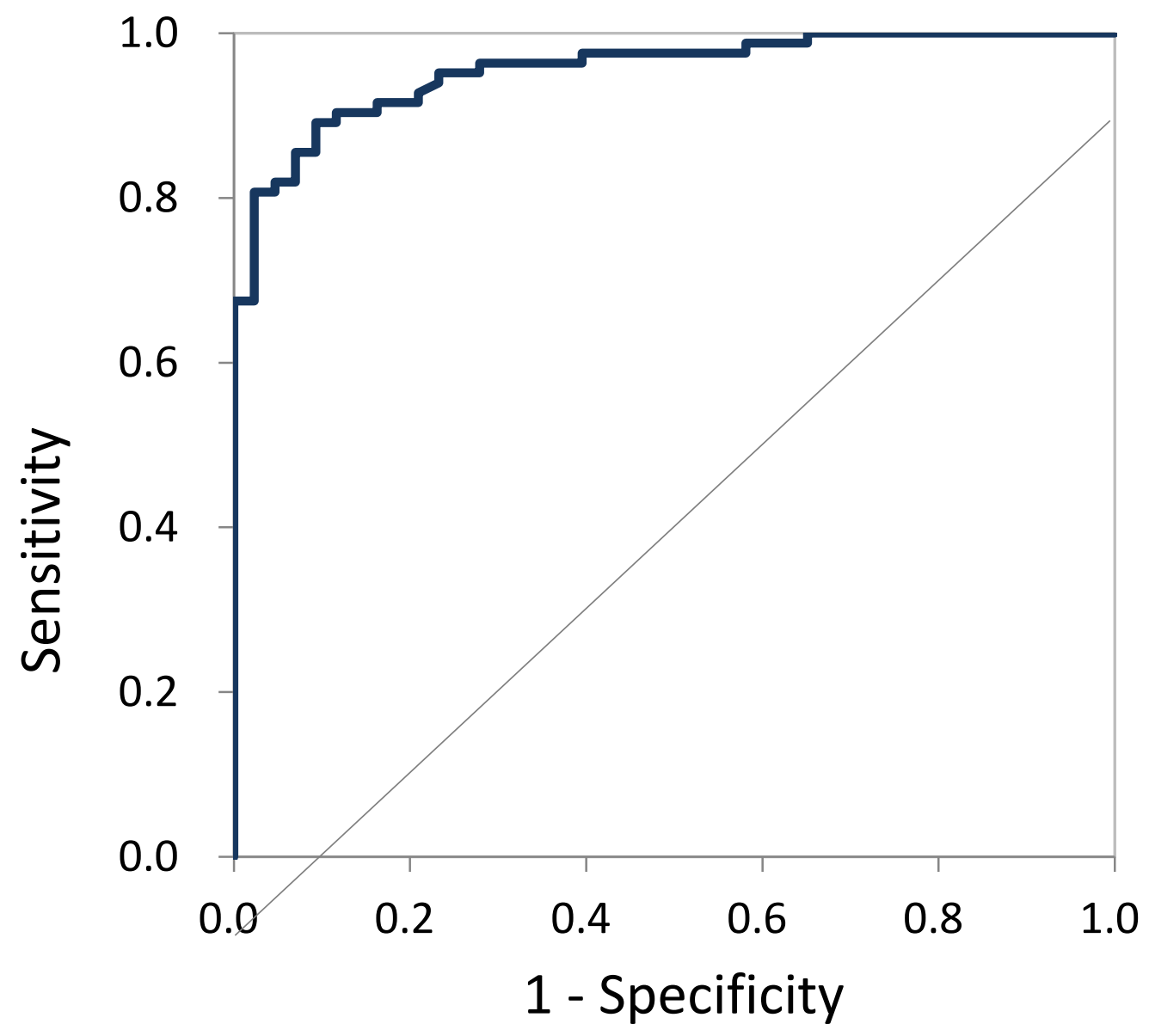

Figure 10. QSM allows accurate assessment of MS lesion enhancement status without Gd injection. QSM isointense predicts Gd-enhancing and QSM hyperintense predicts Gd non-enhancing with an area under the receiver operating characteristic curve of 0.96. (From https://www.ncbi.nlm.nin.gov/pubaned/29365331)

This article is protected by copyright. All rights reserved. 


\section{Figure 10c}

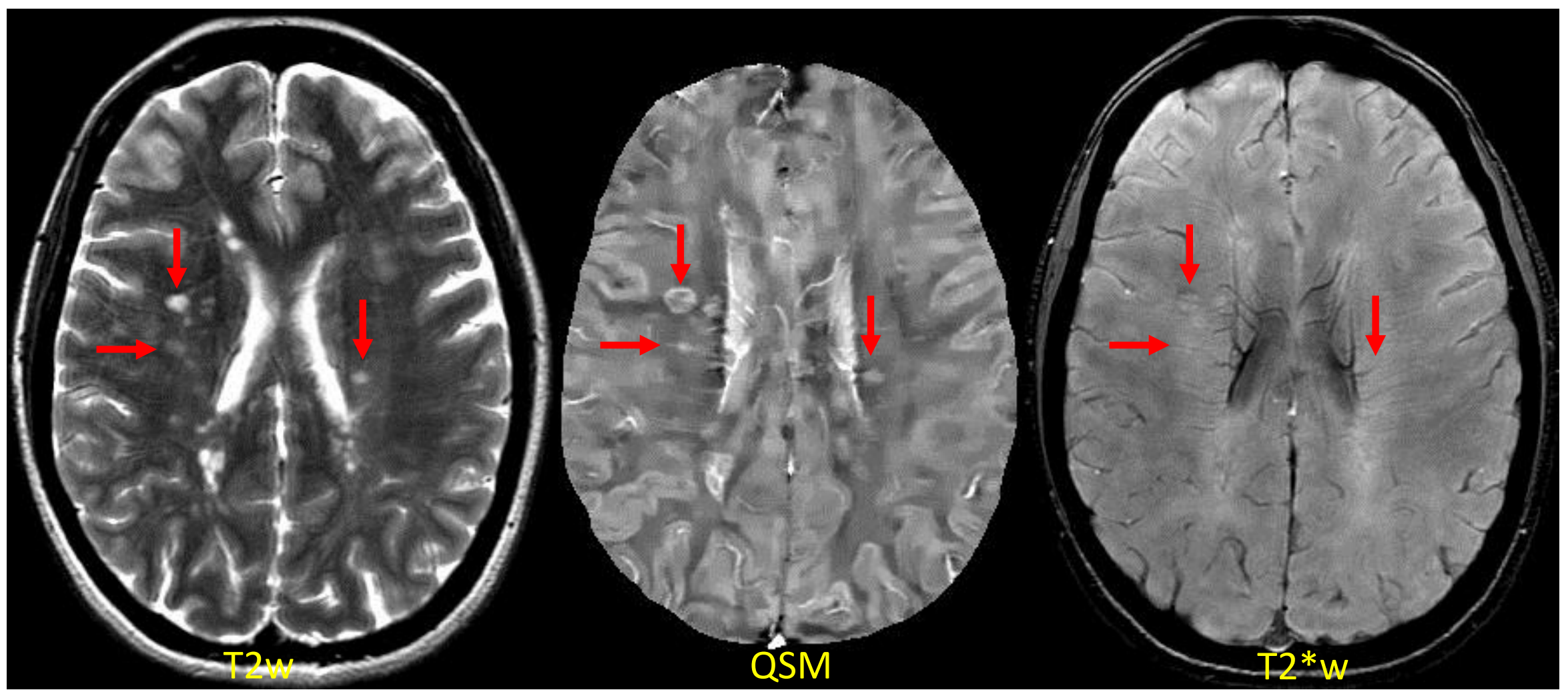

Figure 10. QSM is ideal to depict the central veins in MS lesions (arrows), while T2w cannot depict the central veins, and $\mathrm{T} 2 * \mathrm{w}$ cannot or only very poorly depict the lesions. 


\section{Figure 11}

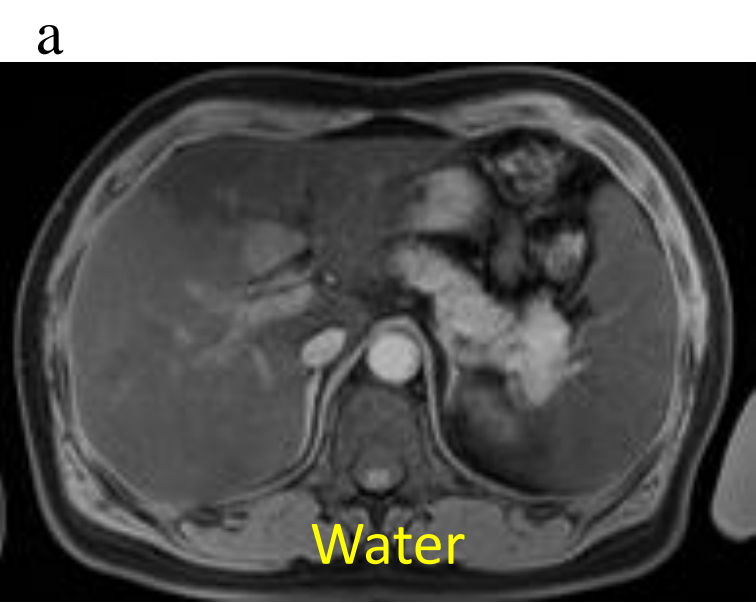

b

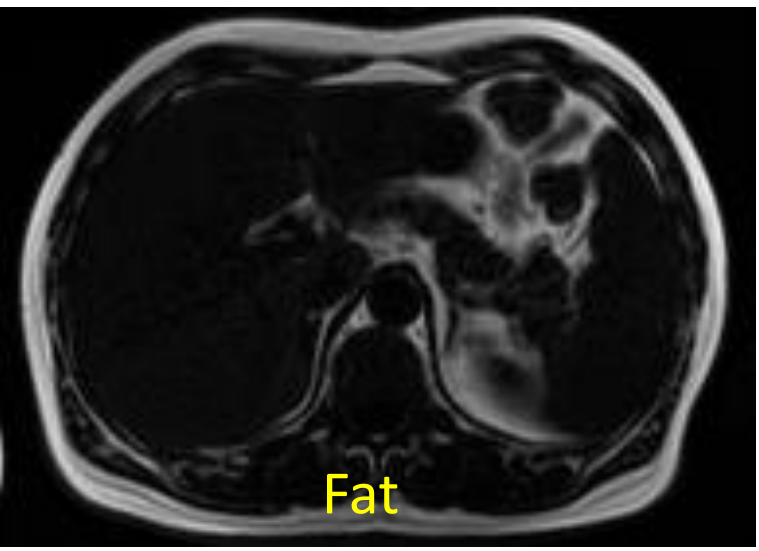

C

d
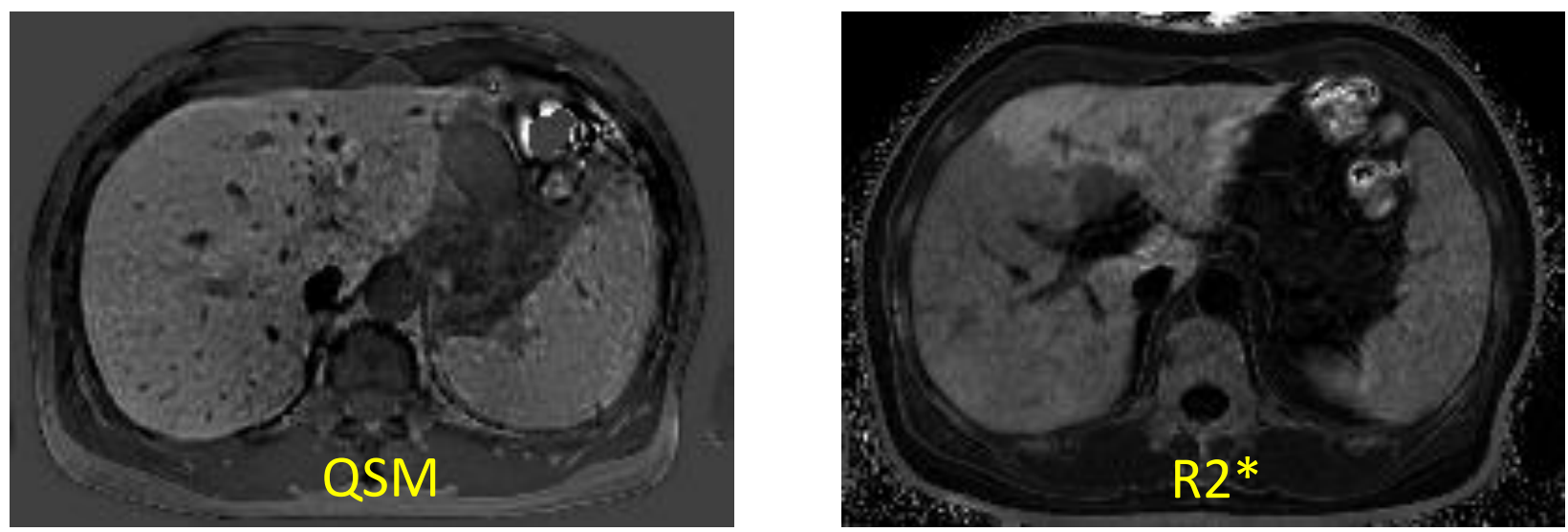

Figure 11. QSM liver iron content quantification. a) Water, b) fat, c) QSM and d) R2* of an axial section of the liver with suspected fibrosis in the medial anterior lobe. Fibrosis did not affect iron quantification by QSM (a) but did affect iron quantification by R2*. QSM overcomes R2* confounding factors, including fibrosis, fat, and edema. Journal of Magnetic Resonance Imaging

This article is protected by copyright. All rights reserved. 


\section{Figure 12}

$\mathrm{a}$

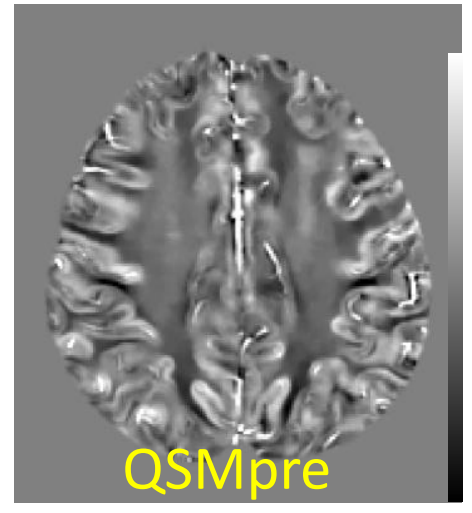

$\mathrm{C}$

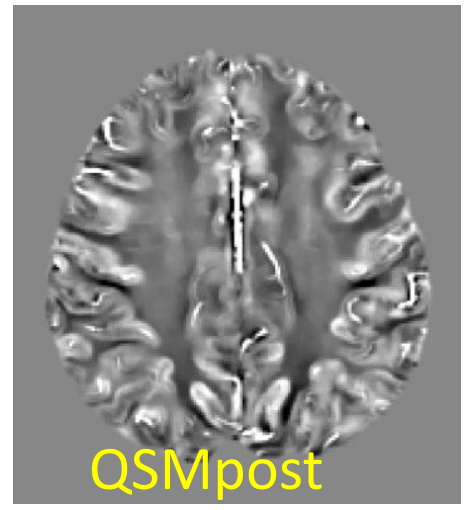

b

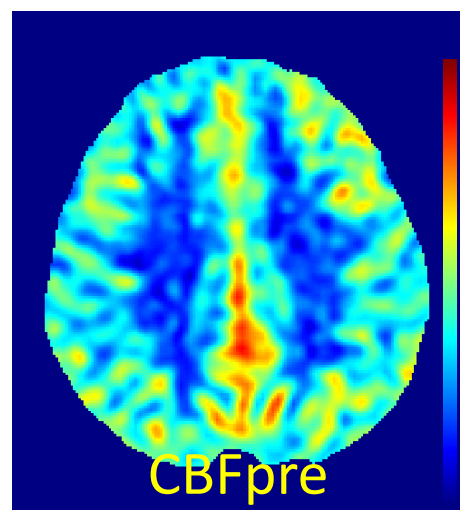

d

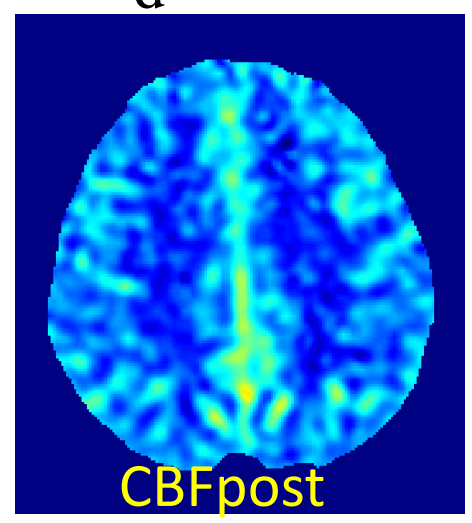

$\mathrm{e}$

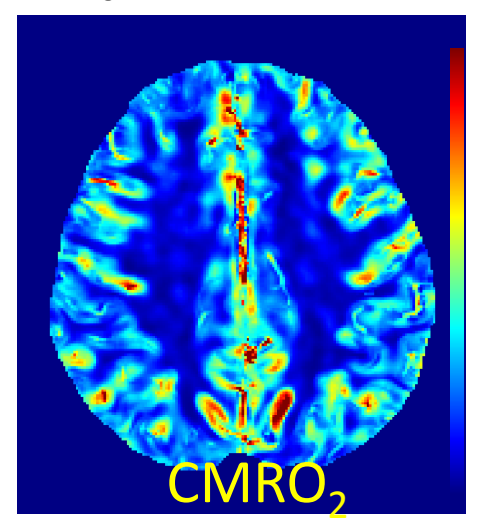

Figure 12. Cerebral metabolic rate of oxygen consumption (CMRO2). a) QSM and b) cerebral blood flow (CBF) were acquired before caffeine challenge, c) QSM and d) CBF after, and e) corresponding CMRO2. QSM is depicted with a gray scale bar [-50,50]ppb, CBF with a color scale bar [0,150]ml/100g/min, and QSM with a color scale bar [0,500] $\mu \mathrm{mol} / 100 \mathrm{~g} / \mathrm{min}$. 


\section{Figure 13}

a

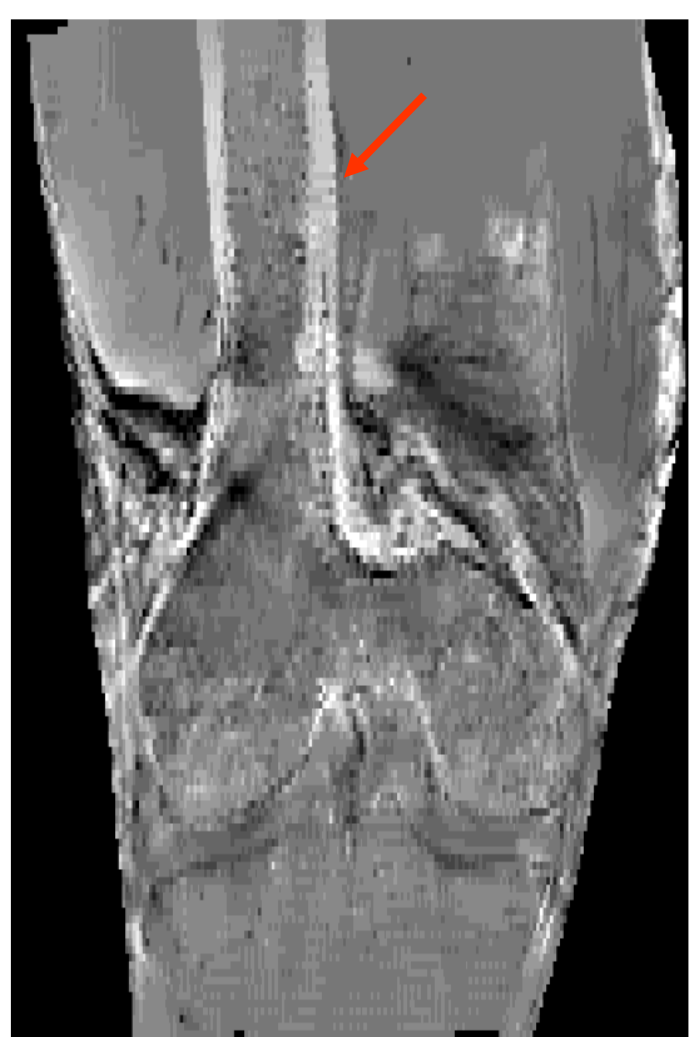

b

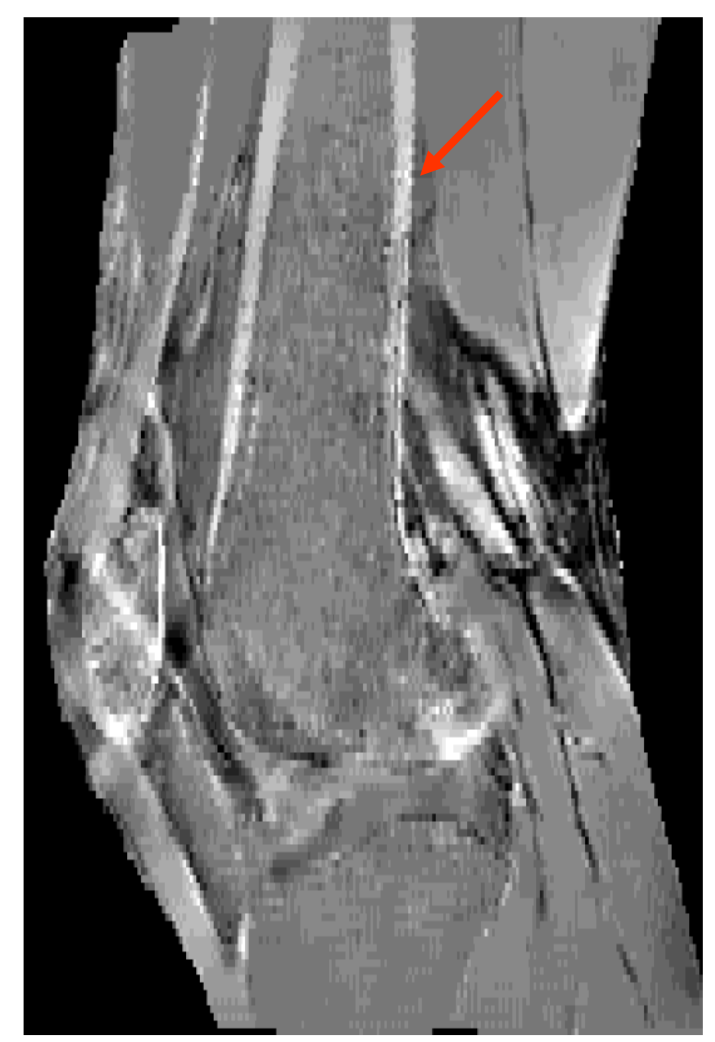

c

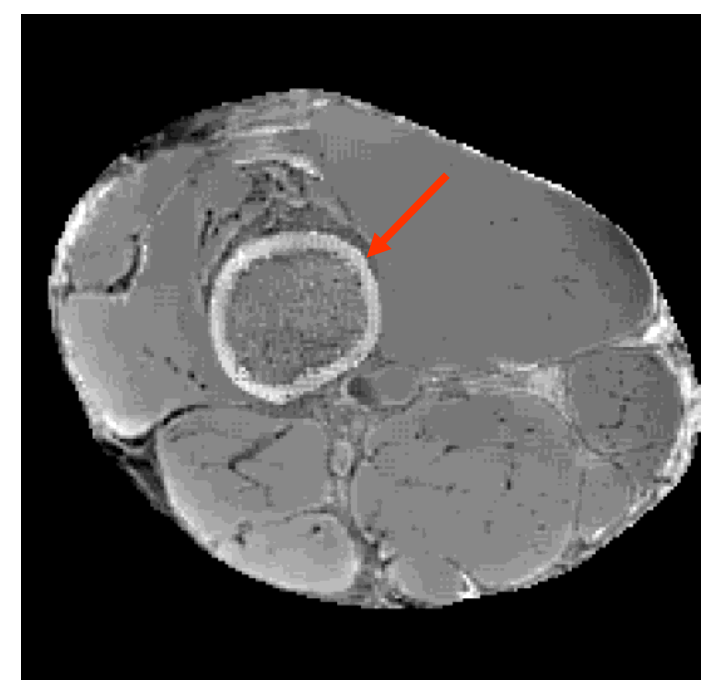

Figure 13. Bone QSM with negative susceptibility depicted as bright. The mineralization in the cortical bone of the femur (arrows) is well captured on QSM as depicted on a a) coronal section, b) sagittal section, and c) axial section. 


\section{University Library}

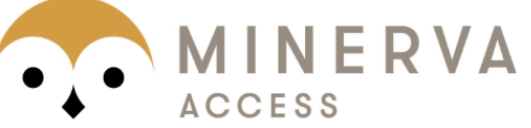

A gateway to Melbourne's research publications

Minerva Access is the Institutional Repository of The University of Melbourne

\section{Author/s:}

Wang, Y;Spincemaille, P;Liu, Z;Dimov, A;Deh, K;Li, J;Zhang, Y;Yao, Y;Gillen, KM;Wilman, AH;Gupta, A;Tsiouris, AJ;Kovanlikaya, I;Chiang, GC-Y;Weinsaft, JW;Tanenbaum, L;Chen, W;Zhu, W;Chang, S;Lou, M;Kopell, BH;Kaplitt, MG;Devos, D;Hirai, T;Huang, X;Korogi, Y;Shtilbans, A;Jahng, G-H;Pelletier, D;Gauthier, SA;Pitt, D;Bush, Al;Brittenham, GM;Prince, MR

Title:

Clinical Quantitative Susceptibility Mapping (QSM): Biometal Imaging and Its Emerging Roles in Patient Care

Date:

2017-10-01

\section{Citation:}

Wang, Y., Spincemaille, P., Liu, Z., Dimov, A., Deh, K., Li, J., Zhang, Y., Yao, Y., Gillen, K. M., Wilman, A. H., Gupta, A., Tsiouris, A. J., Kovanlikaya, I., Chiang, G. C. -Y., Weinsaft, J. W., Tanenbaum, L., Chen, W., Zhu, W., Chang, S. ,.. Prince, M. R. (2017). Clinical Quantitative Susceptibility Mapping (QSM): Biometal Imaging and Its Emerging Roles in Patient Care. JOURNAL OF MAGNETIC RESONANCE IMAGING, 46 (4), pp.951-971. https:// doi.org/10.1002/jmri.25693.

Persistent Link:

http://hdl.handle.net/11343/292630 